\title{
PHYSICAL ORBIT FOR $\lambda$ VIRGINIS AND A TEST OF STELLAR EVOLUTION MODELS
}

\author{
M. Zhao, ${ }^{1}$ J. D. Monnier, ${ }^{1}$ G. Torres,${ }^{2}$ A. F. Boden, ${ }^{3}$ A. Claret, ${ }^{4}$ R. Millan-Gabet, ${ }^{3}$ E. Pedretti, ${ }^{1}$ \\ J.-P. Berger, ${ }^{5}$ W. A. Traub, ${ }^{2}$ F. P. Schloerb, ${ }^{6}$ N. P. Carleton, ${ }^{2}$ P. Kern, ${ }^{5}$ \\ M. G. Lacasse, ${ }^{2}$ F. Malbet, ${ }^{5}$ and K. Perraut ${ }^{5}$ \\ Received 2006 September 11; accepted 2006 December 1
}

\begin{abstract}
The star $\lambda$ Virginis is a well-known double-lined spectroscopic Am binary with the interesting property that both stars are very similar in abundance but one is sharp-lined and the other is broad-lined. We present combined interferometric and spectroscopic studies of $\lambda$ Vir. The small scale of the $\lambda$ Vir orbit $(\sim 20$ mas $)$ is well resolved by the Infrared Optical Telescope Array (IOTA), allowing us to determine its elements, as well as the physical properties of the components, to high accuracy. The masses of the two stars are determined to be 1.897 and $1.721 M_{\odot}$, with $0.7 \%$ and $1.5 \%$ errors, respectively, and the two stars are found to have the same temperature of $8280 \pm 200 \mathrm{~K}$. The accurately determined properties of $\lambda$ Vir allow comparisons between observations and current stellar evolution models, and reasonable matches are found. The best-fit stellar model gives $\lambda$ Vir a subsolar metallicity of $Z=0.0097$ and an age of 935 Myr. The orbital and physical parameters of $\lambda$ Vir also allow us to study its tidal evolution timescales and status. Although atomic diffusion is currently considered to be the most plausible cause of the Am phenomenon, the issue is still being actively debated in the literature. With the present study of the properties and evolutionary status of $\lambda$ Vir, this system is an ideal candidate for further detailed abundance analyses that might shed more light on the source of the chemical anomalies in these A stars.
\end{abstract}

Subject headings: binaries: spectroscopic — binaries: visual — instrumentation: interferometers stars: fundamental parameters - stars: individual ( $\lambda$ Virginis)

Online material: color figures

\section{INTRODUCTION}

Am stars were first recognized by Titus \& Morgan (1940) as a group of stars for which spectral classification is ambiguous. The $\mathrm{Ca}$ II $\mathrm{K}$ lines correspond to earlier types than derived from the Balmer lines, which in turn give earlier types than the metallic lines. Am stars generally have deficient $\mathrm{CNO}$ abundances (e.g., Roby \& Lambert 1990; Sadakane \& Okyudo 1989, etc.), while their iron peak and rare earth elements are generally overabundant (van't Veer-Menneret et al. 1988; Cayrel et al. 1991). Statistical studies (Abt 1961, 2000; Abt \& Morrell 1995) suggest that virtually all Am stars are binaries with projected equatorial rotational velocities less than $120 \mathrm{~km} \mathrm{~s}^{-1}$, and it is the slow rotation that causes the abundance anomalies of Am stars. It is now widely believed that atomic diffusion in slowly rotating stars (e.g., Am and Ap stars) will occur in an outer convection zone so that some elements will be depleted in the atmosphere while others will become overabundant, which partly explains the chemical peculiarity of these stars (Michaud 1980; Richer et al. 1998). Recent progress has been made on atomic diffusion models (Richer et al. 2000), and Michaud et al. (2005) have shown an example study of $o$ Leo indicating that these models can produce abundance anomalies that are consistent with observations. However, the masses they adopted from Griffin (2002) have much larger error bars (more than 20 times larger) than the original determinations of Hummel

\footnotetext{
1 Astronomy Department, University of Michigan, Ann Arbor, MI; mingzhao@ umich.edu.

${ }^{2}$ Harvard-Smithsonian Center for Astrophysics, Cambridge, MA.

3 Michelson Science Center, California Institute of Technology, Pasadena, CA.

4 Instituto de Astrofísica de Andalucía, CSIC, Granada, Spain.

5 Laboratoire d'Astrophysique de Grenoble, Saint Martin d'Heres, France.

6 Department of Astronomy, University of Massachusetts, Amherst, MA.
}

et al. (2001), and no explanation was given for such a large difference. This implies that if the values from Griffin (2002) were wrong, the studies of Michaud et al. (2005) would be affected, and their conclusions might be changed as well. Very recently, BöhmVitense (2006) studied the interaction between Am stars and the interstellar medium, and suggested that the Am phenomenon may be due at least in part to accretion of interstellar material rather than the more popular explanation in terms of atomic diffusion processes. This study challenges the most popular explanation of the Am phenomenon and makes this puzzle more interesting yet still unclear. Although Am stars have been studied intensively since their discovery, only a few of them have well-determined properties. Therefore, in order to address these problems, more precise and accurate measurements of Am stars are required so that more detailed studies can be conducted to help improve our understanding of the role of atomic diffusion and, eventually, the cause of the abundance anomalies in Am stars.

The star $\lambda$ Virginis (HD 125337, HIP 69974, HR 5359; $V=$ $4.523 \mathrm{mag}, H=4.282 \mathrm{mag}$ ) was first reported to be a doublelined spectroscopic binary by Campbell et al. (1911). The two components were classified as metallic-lined A (Am) stars (Cowley et al. 1969; Levato 1975). Early spectroscopic studies estimated its orbital parameters and found a period of 206 days with very low eccentricity ( 0.079) (Colacevich 1941; Abt 1961; Stickland 1975, 1990). Chemical abundance studies (Colacevich 1941; Stickland 1975) suggested the interesting property of $\lambda$ Vir that both stars are very similar in abundance despite their different rotation velocities with the primary being broad-lined (with $v \sin i=35 \mathrm{~km} \mathrm{~s}^{-1}$ ) and the secondary sharp-lined (with $v \sin i=$ $16 \mathrm{~km} \mathrm{~s}^{-1}$ ). The differing rotation rates and the unusual metalliclined nature of the system, as well as the similarity in the abundance of the two components, give us a unique opportunity to test stellar models and study its evolutionary status. 
In this paper, we report the combined interferometric and spectroscopic study of $\lambda$ Vir and the testing of stellar evolution models. The observations span several orbital periods, providing enough orbital coverage and allowing us to deduce the orbital and physical properties of the system precisely. After describing the observations in $\S 2$, we present the orbit determination in $\S 3$, including the discussion of the bandwidth-smearing effect for the interferometric visibilities and biases in closure-phase measurements. We determine its physical properties in $\S 4$ and compare the resulting properties with stellar models in $\S 5$ and tidal evolution theory in $\S 6$. Finally, we give our conclusions and summary in $\S 7$.

\section{OBSERVATIONS}

\subsection{Spectroscopic Observations and Reductions}

The spectroscopic observations of $\lambda$ Vir were conducted at the Harvard-Smithsonian Center for Astrophysics (CfA) between 1982 July and 1991 February, mostly with an echelle spectrograph on the $1.5 \mathrm{~m}$ Wyeth reflector at the Oak Ridge Observatory (Harvard, Massachusetts). A single echelle order was recorded with an intensified Reticon diode array giving a spectral coverage of about $45 \AA$ at a central wavelength of $5188.5 \AA$. The main spectral feature in this region is the $\mathrm{Mg}_{\mathrm{I}} b$ triplet, although there are numerous other metallic lines as well. The resolving power is $\lambda / \Delta \lambda \approx 35,000$. Occasional observations were made also with nearly identical instruments on the $1.5 \mathrm{~m}$ Tillinghast reflector at the F. L. Whipple Observatory (Mount Hopkins, Arizona) and the Multiple Mirror Telescope (also on Mount Hopkins, Arizona), prior to its conversion to a monolithic mirror. A total of $130 \mathrm{spec}-$ tra were collected, with signal-to-noise ratios ( $\mathrm{S} / \mathrm{Ns}$ ) ranging from 20 to about 50 per resolution element of $8.5 \mathrm{~km} \mathrm{~s}^{-1}$.

Radial velocities were derived using TODCOR (Zucker \& Mazeh 1994), a two-dimensional cross-correlation algorithm well suited to our relatively low $\mathrm{S} / \mathrm{N}$ spectra. TODCOR uses two templates, one for each component of the binary, and significantly reduces systematics due to line blending that are often unavoidable in standard one-dimensional cross-correlation techniques (see, e.g., Latham et al. 1996). The templates were selected from a large library of synthetic spectra based on model atmospheres by R. L. Kurucz, ${ }^{7}$ computed for us by Jon Morse (see also Nordström et al. 1994; Latham et al. 2002). These calculated spectra are available for a wide range of effective temperatures $\left(T_{\text {eff }}\right)$, projected rotational velocities $(v \sin i)$, surface gravities $(\log g)$, and metallicities. Experience has shown that radial velocities are largely insensitive to the surface gravity and metallicity adopted for the templates. Consequently, the optimum template for each star was determined from grids of cross-correlations over broad ranges in temperature and rotational velocity, seeking to maximize the average correlation weighted by the strength of each exposure (see Torres et al. 2002). For the surface gravity we adopted the value of $\log g=4.0$ for both stars ( $\operatorname{see} \S 5)$, and for the metallicity we initially adopted the solar composition. However, in view of the metallic-lined nature of the stars we repeated the procedure for a range of metallicities from $[m / H]=-1.0$ to +0.5 in steps of 0.5 dex. We found the best match to the observed spectra for $[m / H]=+0.5$, which is consistent with the enhanced surface abundances expected for these objects. At this metallicity the effective temperatures we derive are $8800 \pm 200 \mathrm{~K}$ for both stars, and the rotational velocities are $v_{1} \sin i=36 \pm 1 \mathrm{~km} \mathrm{~s}^{-1}$ and $v_{2} \sin i=10 \pm 2 \mathrm{~km} \mathrm{~s}^{-1}$ for the primary and secondary, respectively. The rotational velocity estimates are fairly consistent with determinations by other authors: Stickland (1975) reported 35 and $16 \mathrm{~km} \mathrm{~s}^{-1}$ (no un-

${ }^{7}$ Available at http://cfaku5.cfa.harvard.edu. certainties given), and Abt \& Morrell (1995) estimated 31 and $13 \mathrm{~km} \mathrm{~s}^{-1}$, with uncertainties of about $8 \mathrm{~km} \mathrm{~s}^{-1}$. Very rough values without uncertainties were estimated more recently by Shorlin et al. (2002) as $\sim 50$ and $<10 \mathrm{~km} \mathrm{~s}^{-1}$. We discuss the temperature estimates in $\S 4$.

In addition to the radial velocities and stellar parameters, we derived the spectroscopic light ratio following Zucker \& Mazeh (1994). The result, $\ell_{2} / \ell_{1}=0.58 \pm 0.02$, corresponds to the mean wavelength of our observations $(5188.5 \AA)$ and is not far from the visual band.

Due to the narrow wavelength coverage of the CfA spectra there is always the possibility of systematic errors in the velocities, resulting from lines of the stars moving in and out of the spectral window with orbital phase (Latham et al. 1996). Occasionally these errors are significant, and experience has shown that this must be checked on a case-by-case basis (see, e.g., Torres et al. 1997, 2000). For this we performed numerical simulations in which we generated artificial composite spectra by adding together synthetic spectra for the two components, with Doppler shifts appropriate for each actual time of observation, computed from a preliminary orbital solution. The light ratio adopted was that derived above. We then processed these simulated spectra with TODCOR in the same manner as the real spectra, and compared the input and output velocities. Although the differences for $\lambda$ Vir were well under $1 \mathrm{~km} \mathrm{~s}^{-1}$, they are systematic in nature, and we therefore applied them as corrections to the raw velocities for completeness. The final velocities including these corrections are given in Table 1. Similar corrections were derived for the light ratio and are already accounted for in the value reported above.

The stability of the zero point of the velocity system was monitored by means of exposures of the dusk and dawn sky, and small run-to-run corrections were applied in the manner described by Latham et al. (1992). These corrections are also included in Table 1. The accuracy of the CfA velocity system, which is within about $0.14 \mathrm{~km} \mathrm{~s}^{-1}$ of the reference frame defined by minor planets in the solar system, is documented in the previous citation and also by Stefanik et al. (1999) and Latham et al. (2002).

\subsection{Interferometric Observations and Data Reduction}

The interferometric observations of $\lambda$ Vir were carried out using the IOTA (Traub et al. 2003), also at the F. L. Whipple Observatory. IOTA is a three $0.45 \mathrm{~m}$ telescope interferometer array that is movable along its L-shaped southeast and northeast arms, providing several different array configurations and having baselines up to $38 \mathrm{~m}$. Light from each telescope is focused into a singlemode fiber, and the beams from three fibers are split and combined by the "pair-wise" beam combiner Integrated Optics Near-infrared Interferometric Camera (IONIC-3; Berger et al. 2003) to form six fringes. Fringes are temporally scanned by piezo scanners in the delay lines and are then detected by a PICNIC camera (Pedretti et al. 2004). This detection scheme leads to high sensitivities for IOTA ( $\sim 7$ th magnitude at $H$ band; Monnier et al. 2004) and allows for precise measurements of visibilities and closure phases.

The observations reported here were taken in the $H$ band $\left(\lambda_{0}=1.647 \mu \mathrm{m}, \Delta \lambda=0.30 \mu \mathrm{m}\right)$ between 2003 February and 2005 June, spanning four orbital periods (853 days) and covering a broad range of orbital phases, and different array configurations were applied to obtain good $u$ - $v$ coverage. The observations were carried out following the standard procedures (e.g., Monnier et al. 2004), and the observation log is listed in Table 2. In short, $\lambda$ Vir was observed in conjunction with nearby unresolved calibrators (HD 126035, HD 129502, HD 158352) to calibrate the varying system visibilities and closure phases caused by the instrumental response and the effect of atmospheric seeing. Each 
TABLE 1

Radial Velocity of $\lambda V_{\text {IR }}$

\begin{tabular}{|c|c|c|c|}
\hline $\begin{array}{c}\text { HJD } \\
(+2,400,000.5)\end{array}$ & Orbital Phase & $\begin{array}{c}\mathrm{RV}_{1} \\
\left(\mathrm{~km} \mathrm{~s}^{-1}\right)\end{array}$ & $\begin{array}{c}\mathrm{RV}_{2} \\
\left(\mathrm{~km} \mathrm{~s}^{-1}\right)\end{array}$ \\
\hline $45,156.5547 \ldots \ldots \ldots \ldots \ldots \ldots \ldots$ & 0.72 & -31.80 & 18.58 \\
\hline $46,576.6328 \ldots \ldots \ldots \ldots \ldots \ldots .$. & 0.59 & -19.43 & 5.80 \\
\hline $46,576.6758 \ldots \ldots \ldots \ldots \ldots \ldots .$. & 0.59 & -20.03 & 5.50 \\
\hline $46,576.6797 \ldots \ldots \ldots \ldots \ldots \ldots \ldots . .$. & 0.59 & -19.24 & 5.70 \\
\hline $46,597.6406 \ldots \ldots \ldots \ldots \ldots \ldots \ldots . .$. & 0.69 & -30.60 & 16.41 \\
\hline $46,597.6562 \ldots \ldots \ldots \ldots \ldots \ldots \ldots$ & 0.69 & -30.60 & 16.34 \\
\hline $46,597.6641 \ldots \ldots \ldots \ldots \ldots \ldots \ldots$ & 0.69 & -31.93 & 17.30 \\
\hline $46,611.5703 \ldots \ldots \ldots \ldots \ldots \ldots$ & 0.76 & -33.75 & 19.10 \\
\hline $46,613.5664 \ldots \ldots \ldots \ldots \ldots \ldots . .$. & 0.77 & -32.03 & 19.33 \\
\hline $46,633.6016 \ldots \ldots \ldots \ldots \ldots \ldots .$. & 0.86 & -26.78 & 13.07 \\
\hline $46,635.5430 \ldots \ldots \ldots \ldots \ldots \ldots \ldots$ & 0.87 & -27.06 & 11.62 \\
\hline $46,636.5430 \ldots \ldots \ldots \ldots \ldots \ldots \ldots$ & 0.88 & -28.03 & 10.66 \\
\hline $46,640.5586 \ldots \ldots \ldots \ldots \ldots \ldots \ldots$ & 0.90 & -23.66 & 9.12 \\
\hline $46,809.9688 \ldots \ldots \ldots \ldots \ldots \ldots \ldots$ & 0.72 & -32.51 & 18.19 \\
\hline 46,819.9570 & 0.77 & -34.08 & 19.31 \\
\hline $46,896.7656 \ldots \ldots \ldots \ldots \ldots \ldots$. & 0.14 & 14.56 & -30.78 \\
\hline $46,918.7227 \ldots \ldots \ldots \ldots \ldots \ldots . .$. & 0.24 & 17.31 & -34.79 \\
\hline $46,924.6719 \ldots \ldots \ldots \ldots \ldots \ldots \ldots$ & 0.27 & 16.75 & -33.91 \\
\hline $46,938.6758 \ldots \ldots \ldots \ldots \ldots \ldots$ & 0.34 & 12.25 & -28.73 \\
\hline $46,953.6094 \ldots \ldots \ldots \ldots \ldots \ldots$ & 0.41 & 1.66 & -20.67 \\
\hline $47,197.9453 \ldots \ldots \ldots \ldots \ldots \ldots .$. & 0.59 & -19.45 & 6.86 \\
\hline $47,206.0508 \ldots \ldots \ldots \ldots \ldots \ldots . .$. & 0.63 & -25.53 & 11.80 \\
\hline $47,218.9141 \ldots \ldots \ldots \ldots \ldots \ldots \ldots$ & 0.70 & -30.68 & 18.07 \\
\hline $47,222.8750 \ldots \ldots \ldots \ldots \ldots$ & 0.71 & -32.75 & 16.99 \\
\hline $47,226.8438 \ldots \ldots \ldots \ldots \ldots \ldots .$. & 0.73 & -30.44 & 20.05 \\
\hline $47,320.7148 \ldots \ldots \ldots \ldots \ldots \ldots$ & 0.19 & 17.45 & -34.27 \\
\hline $47,568.8828 \ldots \ldots \ldots \ldots \ldots \ldots . .$. & 0.39 & 5.82 & -23.65 \\
\hline $47,569.9688 \ldots \ldots \ldots \ldots \ldots \ldots .$. & 0.39 & 5.08 & -22.15 \\
\hline $47,570.9414 \ldots \ldots \ldots \ldots \ldots \ldots . .$. & 0.40 & 2.87 & -22.46 \\
\hline $47,574.9297 \ldots \ldots \ldots \ldots \ldots \ldots . .$. & 0.42 & 0.86 & -20.01 \\
\hline $47,575.9141 \ldots \ldots \ldots \ldots \ldots \ldots$ & 0.42 & -0.60 & -19.07 \\
\hline $47,583.8047 \ldots \ldots \ldots \ldots \ldots \ldots$ & 0.46 & -6.53 & -13.33 \\
\hline $47,585.9062 \ldots \ldots \ldots \ldots \ldots \ldots . .$. & 0.47 & -6.35 & -11.88 \\
\hline $47,586.8359 \ldots \ldots \ldots \ldots \ldots \ldots . .$. & 0.48 & -6.70 & -11.02 \\
\hline $47,587.8633 \ldots \ldots \ldots \ldots \ldots \ldots .$. & 0.48 & -9.53 & -10.34 \\
\hline $47,598.8789 \ldots \ldots \ldots \ldots \ldots \ldots \ldots$ & 0.53 & -14.02 & -2.58 \\
\hline $47,602.8516 \ldots \ldots \ldots \ldots \ldots \ldots \ldots$ & 0.55 & -15.38 & 0.74 \\
\hline $47,607.8242 \ldots \ldots \ldots \ldots \ldots \ldots \ldots$ & 0.58 & -18.86 & 4.20 \\
\hline $47,608.7930 \ldots \ldots \ldots \ldots \ldots \ldots . .$. & 0.58 & -21.07 & 4.90 \\
\hline $47,612.8398 \ldots \ldots \ldots \ldots \ldots \ldots \ldots$ & 0.60 & -21.97 & 8.06 \\
\hline $47,613.7578 \ldots \ldots \ldots \ldots \ldots \ldots$ & 0.61 & -23.18 & 8.32 \\
\hline $47,628.7344 \ldots \ldots \ldots \ldots \ldots . .$. & 0.68 & -29.95 & 16.34 \\
\hline $47,640.7031 \ldots \ldots \ldots \ldots \ldots \ldots$ & 0.74 & -32.58 & 18.24 \\
\hline $47,641.8555 \ldots \ldots \ldots \ldots \ldots \ldots$ & 0.74 & -30.13 & 19.15 \\
\hline $47,642.7695 \ldots \ldots \ldots \ldots \ldots \ldots . .$. & 0.75 & -33.43 & 18.76 \\
\hline $47,643.7383 \ldots \ldots \ldots \ldots \ldots \ldots \ldots$ & 0.75 & -32.53 & 18.59 \\
\hline $47,644.7266 \ldots \ldots \ldots \ldots \ldots \ldots$ & 0.76 & -33.16 & 18.78 \\
\hline $47,661.7305 \ldots \ldots \ldots \ldots \ldots \ldots$ & 0.84 & -30.64 & 15.75 \\
\hline $47,662.6680 \ldots \ldots \ldots \ldots \ldots \ldots .$. & 0.84 & -30.49 & 15.06 \\
\hline 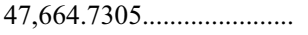 & 0.85 & -30.27 & 14.10 \\
\hline $47,665.6602 \ldots \ldots \ldots \ldots \ldots \ldots \ldots$ & 0.86 & -26.94 & 14.82 \\
\hline $47,674.5586 \ldots \ldots \ldots \ldots \ldots \ldots . .$. & 0.90 & -24.69 & 8.50 \\
\hline $47,675.6406 \ldots \ldots \ldots \ldots \ldots \ldots$ & 0.91 & -24.16 & 7.88 \\
\hline $47,676.6641 \ldots \ldots \ldots \ldots \ldots \ldots . .$. & 0.91 & -21.57 & 7.61 \\
\hline $47,688.5938 \ldots \ldots \ldots \ldots \ldots \ldots . .$. & 0.97 & -14.10 & -2.76 \\
\hline $47,689.7031 \ldots \ldots \ldots \ldots \ldots \ldots . .$. & 0.97 & -12.11 & -3.75 \\
\hline $47,693.6289 \ldots \ldots \ldots \ldots \ldots . .$. & 0.99 & -11.58 & -8.51 \\
\hline $47,698.6328 \ldots \ldots \ldots \ldots \ldots \ldots . .$. & 0.02 & -7.05 & -12.13 \\
\hline $47,702.6523 \ldots \ldots \ldots \ldots \ldots \ldots . .$. & 0.04 & -3.51 & -15.23 \\
\hline $47,723.5664 \ldots \ldots \ldots \ldots \ldots \ldots \ldots$ & 0.14 & 11.06 & -31.07 \\
\hline $47,730.5547 \ldots \ldots \ldots \ldots \ldots \ldots \ldots$ & 0.17 & 14.62 & -33.99 \\
\hline $47,763.5078 \ldots \ldots \ldots \ldots \ldots \ldots \ldots$ & 0.33 & 12.28 & -29.11 \\
\hline 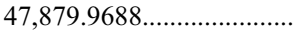 & 0.89 & -24.54 & 9.80 \\
\hline 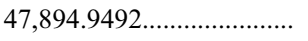 & 0.97 & -14.83 & -2.20 \\
\hline $47,895.9453 \ldots \ldots \ldots \ldots \ldots \ldots \ldots$ & 0.97 & -12.76 & -3.57 \\
\hline
\end{tabular}

TABLE 1-Continued

\begin{tabular}{|c|c|c|c|}
\hline $\begin{array}{c}\text { HJD } \\
(+2,400,000.5)\end{array}$ & Orbital Phase & $\begin{array}{c}\mathrm{RV}_{1} \\
\left(\mathrm{~km} \mathrm{~s}^{-1}\right)\end{array}$ & $\begin{array}{c}\mathrm{RV}_{2} \\
\left(\mathrm{~km} \mathrm{~s}^{-1}\right)\end{array}$ \\
\hline $47,898.9492 \ldots \ldots$ & 0.99 & -12.30 & -6.20 \\
\hline 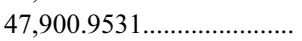 & 0.99 & -10.97 & -9.37 \\
\hline 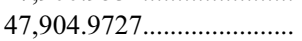 & 0.01 & -6.57 & -12.07 \\
\hline $47,908.9570 \ldots \ldots \ldots \ldots \ldots \ldots \ldots \ldots$ & 0.03 & -5.09 & -15.27 \\
\hline $47,910.9453 \ldots \ldots \ldots \ldots \ldots \ldots \ldots$ & 0.04 & -3.03 & -16.64 \\
\hline $47,922.9492 \ldots \ldots \ldots \ldots \ldots \ldots \ldots$ & 0.10 & 7.00 & -27.02 \\
\hline $47,928.8906 \ldots \ldots \ldots \ldots \ldots \ldots \ldots$ & 0.13 & 10.71 & -30.08 \\
\hline $47,930.9648 \ldots \ldots \ldots \ldots \ldots \ldots \ldots$ & 0.14 & 13.31 & -30.51 \\
\hline $47,931.9258 \ldots \ldots \ldots \ldots \ldots \ldots \ldots$ & 0.14 & 14.32 & -31.36 \\
\hline $47,933.9453 \ldots \ldots \ldots \ldots \ldots \ldots \ldots$ & 0.15 & 12.59 & -32.76 \\
\hline 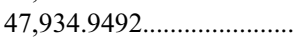 & 0.16 & 15.58 & -33.16 \\
\hline $47,935.8750 \ldots \ldots \ldots \ldots \ldots \ldots \ldots$ & 0.16 & 15.39 & -33.56 \\
\hline 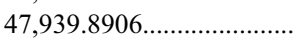 & 0.18 & 15.06 & -34.87 \\
\hline 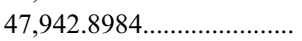 & 0.20 & 16.54 & -35.06 \\
\hline 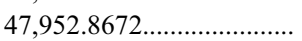 & 0.25 & 16.34 & -35.10 \\
\hline 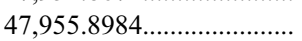 & 0.26 & 16.30 & -34.90 \\
\hline 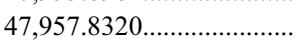 & 0.27 & 14.77 & -35.07 \\
\hline $47,958.9414 \ldots \ldots \ldots \ldots \ldots$ & 0.28 & 16.70 & -34.97 \\
\hline $47,959.8477 \ldots \ldots \ldots \ldots \ldots$ & 0.28 & 15.94 & -33.16 \\
\hline $47,960.8672 \ldots \ldots \ldots \ldots \ldots \ldots$ & 0.28 & 15.77 & -33.39 \\
\hline $47,963.8711 \ldots \ldots \ldots \ldots \ldots \ldots \ldots$ & 0.30 & 14.87 & -33.14 \\
\hline 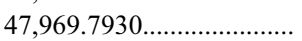 & 0.33 & 13.14 & -30.06 \\
\hline $47,989.7422 \ldots \ldots \ldots \ldots \ldots \ldots \ldots$ & 0.42 & -1.21 & -17.35 \\
\hline $47,990.7617 \ldots \ldots \ldots \ldots \ldots \ldots \ldots$ & 0.43 & -2.36 & -17.36 \\
\hline 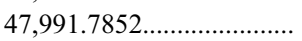 & 0.43 & -3.27 & -16.77 \\
\hline $47,994.7852 \ldots \ldots \ldots \ldots \ldots \ldots \ldots$ & 0.45 & -3.84 & -14.83 \\
\hline 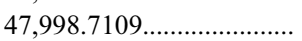 & 0.47 & -5.63 & -11.45 \\
\hline $47,999.7344 \ldots \ldots \ldots \ldots \ldots \ldots \ldots$ & 0.47 & -5.93 & -10.69 \\
\hline $48,000.7148 \ldots \ldots \ldots \ldots \ldots \ldots \ldots$ & 0.48 & -6.97 & -10.60 \\
\hline $48,001.7500 \ldots \ldots \ldots \ldots \ldots \ldots \ldots$ & 0.48 & -10.20 & -9.33 \\
\hline $48,021.6758 \ldots \ldots \ldots \ldots \ldots \ldots \ldots$ & 0.58 & -19.67 & 3.84 \\
\hline 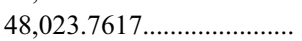 & 0.59 & -23.76 & 5.77 \\
\hline $48,026.6797 \ldots \ldots \ldots \ldots \ldots$ & 0.60 & -22.28 & 7.47 \\
\hline $48,027.6562 \ldots \ldots \ldots \ldots \ldots \ldots \ldots$ & 0.61 & -21.28 & 8.32 \\
\hline $48,042.6328 \ldots \ldots \ldots \ldots \ldots \ldots \ldots$ & 0.68 & -29.91 & 16.44 \\
\hline $48,044.7461 \ldots \ldots \ldots \ldots \ldots \ldots \ldots$ & 0.69 & -29.98 & 17.49 \\
\hline $48,050.6172 \ldots \ldots \ldots \ldots \ldots \ldots \ldots$ & 0.72 & -32.79 & 18.73 \\
\hline $48,052.5742 \ldots \ldots \ldots \ldots \ldots \ldots \ldots$ & 0.73 & -33.89 & 18.41 \\
\hline 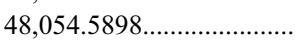 & 0.74 & -32.81 & 19.02 \\
\hline 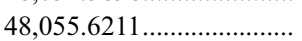 & 0.74 & -34.69 & 18.90 \\
\hline $48,057.6484 \ldots \ldots \ldots \ldots \ldots \ldots \ldots$ & 0.75 & -34.80 & 18.49 \\
\hline $48,058.6992 \ldots \ldots \ldots \ldots \ldots \ldots \ldots$ & 0.76 & -34.14 & 19.76 \\
\hline 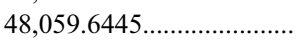 & 0.76 & -35.64 & 17.99 \\
\hline 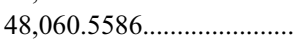 & 0.77 & -32.41 & 19.65 \\
\hline 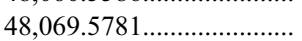 & 0.81 & -31.88 & 17.72 \\
\hline 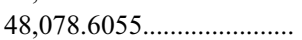 & 0.85 & -28.72 & 14.35 \\
\hline 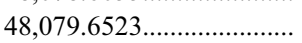 & 0.86 & -26.86 & 15.33 \\
\hline $48,082.5820 \ldots \ldots \ldots \ldots \ldots \ldots \ldots$ & 0.87 & -26.60 & 12.51 \\
\hline $48,084.5625 \ldots \ldots \ldots \ldots \ldots \ldots \ldots$ & 0.88 & -26.50 & 10.60 \\
\hline 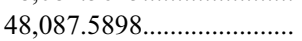 & 0.90 & -25.65 & 8.99 \\
\hline $48,088.5781 \ldots \ldots \ldots \ldots \ldots \ldots$ & 0.90 & -22.51 & 8.12 \\
\hline $48,100.5391 \ldots \ldots \ldots \ldots \ldots \ldots \ldots \ldots$ & 0.96 & -14.22 & -1.31 \\
\hline 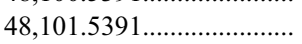 & 0.97 & -14.20 & -3.01 \\
\hline $48,102.5781 \ldots \ldots \ldots \ldots \ldots \ldots$ & 0.97 & -11.95 & -3.36 \\
\hline 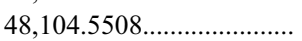 & 0.98 & -11.71 & -6.25 \\
\hline 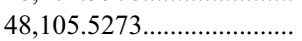 & 0.98 & -10.52 & -6.74 \\
\hline $48,106.5273 \ldots \ldots \ldots \ldots \ldots \ldots \ldots$ & 0.99 & -11.12 & -7.38 \\
\hline $48,108.5312 \ldots \ldots \ldots \ldots \ldots \ldots \ldots$ & 1.00 & -8.76 & -8.89 \\
\hline $48,116.5391 \ldots \ldots \ldots \ldots \ldots \ldots \ldots$ & 0.04 & -0.66 & -15.99 \\
\hline $48,280.9727 \ldots \ldots \ldots \ldots \ldots \ldots \ldots$ & 0.83 & -31.12 & 16.27 \\
\hline $48,281.9727 \ldots \ldots \ldots \ldots \ldots \ldots \ldots$ & 0.84 & -30.50 & 15.95 \\
\hline $48,283.9688 \ldots \ldots \ldots \ldots \ldots \ldots \ldots$ & 0.85 & -30.04 & 15.62 \\
\hline 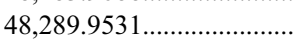 & 0.88 & -26.94 & 11.90 \\
\hline 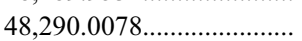 & 0.88 & -28.96 & 11.94 \\
\hline $48,291.8945 \ldots \ldots \ldots \ldots \ldots \ldots \ldots$ & 0.89 & -26.25 & 11.06 \\
\hline
\end{tabular}


TABLE 2

IOTA OBSERVING LOG OF $\lambda$ VIR

\begin{tabular}{|c|c|c|}
\hline $\begin{array}{l}\text { Date }^{\mathrm{a}} \\
\text { (UT) }\end{array}$ & Interferometer Configuration $^{\mathrm{b}}$ & Calibrator Names \\
\hline 2003 Feb 16, 17........................... & A35 B05 C10 & $\begin{array}{l}\text { HD } 126035\left(\mathrm{G} 7 \text { III, } 0.78 \pm 0.24 \mathrm{mas}^{\mathrm{c}}\right) \\
\text { HD } 129502(\mathrm{~F} 2 \text { III, } 1.20 \pm 0.22 \mathrm{mas})\end{array}$ \\
\hline 2003 Feb $20-23 \ldots$ & A25 B05 C10 & HD 126035 \\
\hline 2003 Mar 21 & A 35 B07 C25 & HD 126035 \\
\hline 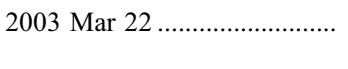 & A35 B07 C10 & $\begin{array}{l}\text { HD } 126035 \\
\text { HD } 158352(\text { A8 V, } 0.44 \pm 0.10 \mathrm{mas})\end{array}$ \\
\hline 2003 Mar 23, 24 & A35 B15 C10 & HD 126035, HD 158352 \\
\hline 2003 Jun $12,14-16 \ldots \ldots \ldots \ldots \ldots . . . .$. & $\mathrm{A} 35 \mathrm{~B} 15 \mathrm{C} 10$ & HD 126035 \\
\hline 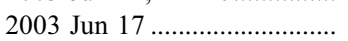 & $\mathrm{A} 35 \mathrm{~B} 15 \mathrm{C} 10$ & HD 126035 \\
\hline 2004 Mar $16-21$ & A35 B15 C10 & HD 126035 \\
\hline 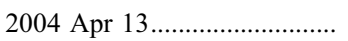 & $\mathrm{A} 35 \mathrm{~B} 15 \mathrm{C} 10$ & HD 129502 \\
\hline 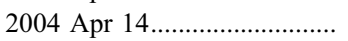 & $\mathrm{A} 35 \mathrm{~B} 15 \mathrm{C} 10$ & HD 126035, HD 129502 \\
\hline 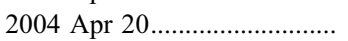 & $\mathrm{A} 35 \mathrm{~B} 15 \mathrm{C} 10$ & HD 126035, HD 158352 \\
\hline 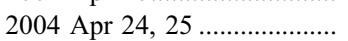 & $\mathrm{A} 35 \mathrm{~B} 15 \mathrm{C} 10$ & HD 126035 \\
\hline 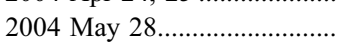 & $\mathrm{A} 35 \mathrm{~B} 15 \mathrm{C} 10$ & HD 126035 \\
\hline 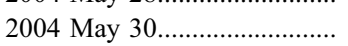 & $\mathrm{A} 35 \mathrm{~B} 15 \mathrm{C} 10$ & HD 126035, HD 129502, HD 158352 \\
\hline 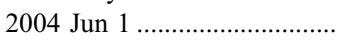 & $\mathrm{A} 35 \mathrm{~B} 15 \mathrm{C} 10$ & HD 126035; HD 158352 \\
\hline 2004 Jun $2-7 \ldots \ldots \ldots \ldots \ldots \ldots$ & A35 B15 C10 & HD 126035 \\
\hline 2005 Jun $14-18 \ldots \ldots \ldots \ldots \ldots \ldots \ldots . .$. & A35 B15 C10 & HD 126035 \\
\hline
\end{tabular}

a Scan mode 1 before 2003 June 16: A: fixed, B: $\Delta X=50.8 \mu \mathrm{m}$, and C: $\Delta X=25.4 \mu \mathrm{m}$; scan mode 2 after 2003 June 16: A: fixed, B: $\Delta X=25.4 \mu \mathrm{m}$, and C: $\Delta X=-25.4 \mu \mathrm{m}$.

${ }^{\mathrm{b}}$ Configuration refers to the location of telescopes A, B, and C on the northeast, southeast, and northeast arms, respectively; see Traub et al. (2003) for more details.

${ }^{\mathrm{c}}$ Uniform disk diameters of the calibrators are generally estimated using getCal, an SED-fitting routine maintained and distributed by the Michelson Science Center.

single observation typically consists of 200 scans within $\sim 4$ minutes, followed by calibration measurements of the background and individual response of each telescope. Two different piezo scan modes were used for different observing runs (see Table 2): mode one before 2003 June 17 (telescope A fixed, telescope B scan range: $50.8 \mu \mathrm{m}$, telescope $\mathrm{C}$ scan range: $25.4 \mu \mathrm{m}$ ) and mode two thereafter (telescope A fixed, telescope B scan range: $25.4 \mu \mathrm{m}$, telescope $\mathrm{C}$ scan range: $-25.4 \mu \mathrm{m}$ ). The effect of different scanning modes is discussed later in the Appendix.

Reduction of the squared visibilities $\left(V^{2}\right)$ and the closure phases was carried out using established IDL routines described by Monnier et al. $(2004,2006)$. In short, we measure the power spectrum of each interferogram, which is proportional to the broadband $V^{2}$ (see Coude du Foresto et al. [1997] for an outline of the method), and correct for intensity fluctuations, as well as bias terms that stem from read noise, background noise, etc. The variable flux ratios of each baseline are calibrated using a flux transfer matrix (Monnier et al. 2006). Measurement errors are obtained from the scatter of the data and are then combined with calibration errors. The calibration error, established statistically from the datafitting procedures (see $\S 4$ ), is $\sim 2 \%$ for $V^{2}$, corresponding to $1 \%$ error in the visibility. In order to measure the closure phases, a realtime fringe-tracking algorithm (Pedretti et al. 2005) was applied to ensure that the interferograms are detected simultaneously in nearly all baselines (at least two are detected if fringes in the third baseline is weak). The closure phases are then obtained by calculating and averaging the bispectrum (triple product) in complex space, with the frequencies of each triple product closed, i.e., $\nu_{\mathrm{AB}}+\nu_{\mathrm{BC}}+\nu_{\mathrm{CA}}=0$ (Baldwin et al. 1996). The instrumental closure-phase offset $\left(\leq 0.5^{\circ}\right.$; Monnier et al. 2006) is calibrated by using unresolved calibrators listed in the observation log. The calibration errors of the closure phases are dominated by fluctuations that result from extra optical path differences (OPDs) caused by the atmospheric piston fluctuations. We discuss this effect in the Appendix and the error estimation for the closure phases in $\S 3.2$.

\section{ORBIT DETERMINATION}

\subsection{Bandwidth-Smearing Effect of $V^{2}$}

Interferometric measurements use a finite range of bandwidth. The resulting fringe packets thus suffer a modulation in the amplitude due to the overlap of fringes with different wavelengths, especially at the edges of the packets. For binary stars, the observed interferogram results from the interference of two fringe packets with an interferometric delay of $\boldsymbol{B} \cdot \boldsymbol{\rho}$ due to the binary separation [where $\boldsymbol{B}$ is the projected baseline vector $\left(B_{x}, B_{y}\right)$ in meters and $\rho$ is the angular separation $(a, b)$ of the binary in units of radians]. Because the two fringe packets are modulated by bandwidth smearing, the resulting observed interferogram is also affected by this, causing significant systematic errors to the measured visibilities and closure phases. This effect is pronounced for broadband filters such as the $H$-band filter of the IOTA PICNIC camera. Our preliminary binary modeling indicated a poor fit to the squared visibilities and the closure phases, evidenced by a large reduced $\chi^{2}\left(\chi_{\nu}^{2}\right)$. Therefore, before we determined the orbit of $\lambda$ Vir, we first investigated the influence of bandwidth smearing on our data.

The standard monochromatic squared visibility of a binary can be written as

$$
V^{2}=\frac{\left|V_{1}\right|^{2}+r^{2}\left|V_{2}\right|^{2}+2 r\left|V_{1}\right|\left|V_{2}\right| \cos [(2 \pi / \lambda) \boldsymbol{B} \cdot \boldsymbol{\rho}]}{(1+r)^{2}},
$$

where $r$ is the flux ratio, and $V_{1}$ and $V_{2}$ are the visibilities of the primary and the secondary, respectively (Boden 2000). For the case of IOTA IONIC-3, in which we measure the power spectrum 

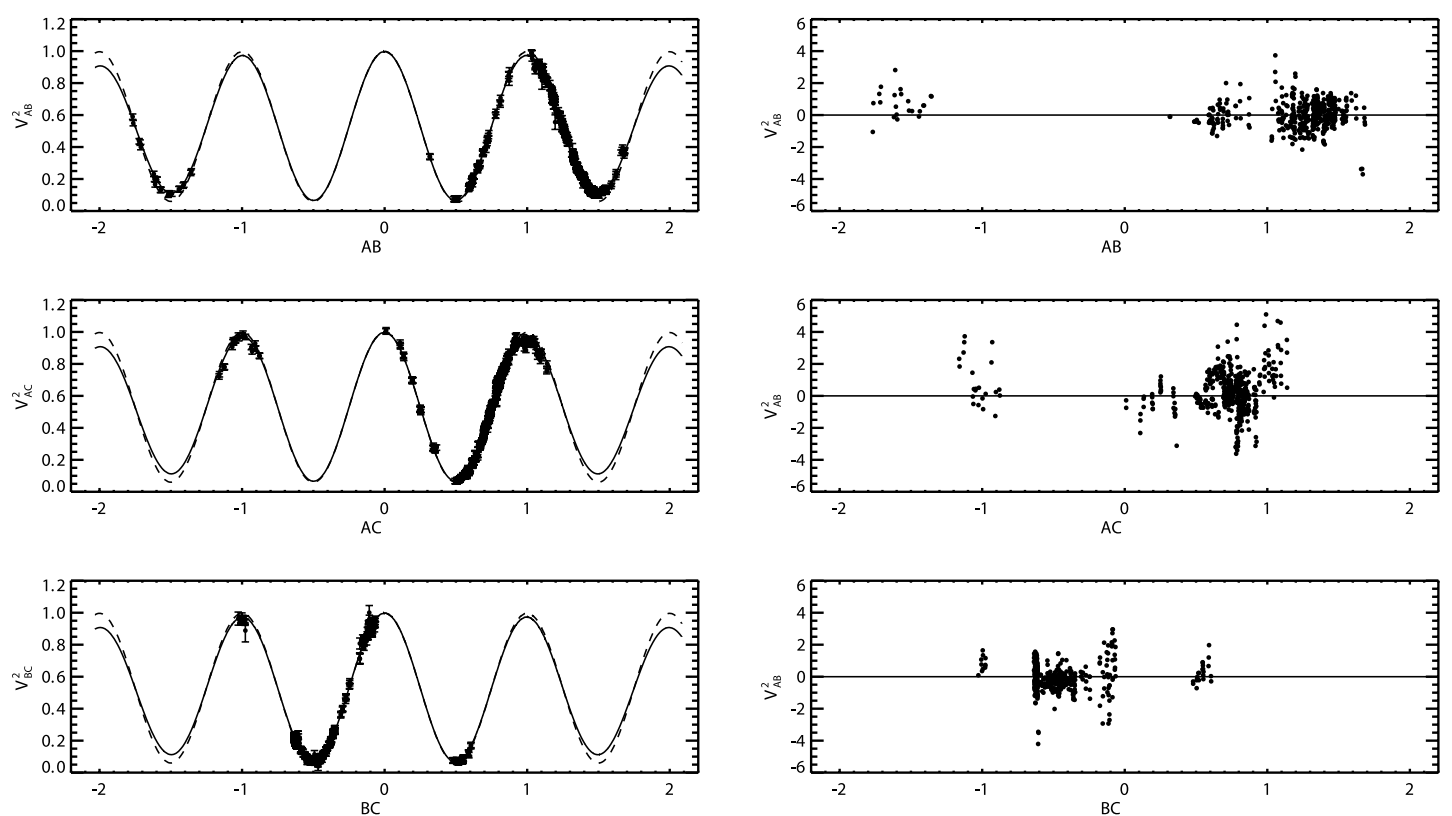

FIG. 1. - The $V^{2}$ for three IOTA baselines vs. interferometric delay $(\boldsymbol{B} \cdot \boldsymbol{\rho})$ in units of wavelengths. The dashed lines indicate the original squared-visibility model with no bandwidth-smearing correction, while the solid lines show the models corrected for bandwidth smearing. The $V^{2}$ data are overplotted with error bars of $1 \sigma$. The corresponding normalized residuals for the corrected model (i.e., residual/error) are shown in the right panels for the three baselines.

of the interferogram to determine the broadband squared visibility $^{8}$ (see, e.g., Coude du Foresto et al. 1997), we integrate the squared visibility over the whole bandpass and subtract equation (1) from it to obtain the difference between the polychromatic and the monochromatic squared visibilities:

$$
\begin{aligned}
\Delta V^{2} & =V_{\mathrm{BS}}^{2}-V^{2} \\
& =\frac{2 r\left|V_{1}\right|\left|V_{2}\right| \cos (2 \pi \delta)\left[\exp \left(-\delta^{2} / 2 f^{2}\right)-1\right]}{(1+r)^{2}},
\end{aligned}
$$

where

$$
f=\frac{\lambda \beta}{\Delta \lambda \sqrt{8 \ln 2}} .
$$

We used a Gaussian envelope function, $\exp \left(-\delta^{2} / 2 f^{2}\right)$, to approximate the modulation of the interferogram, where $\delta=(\boldsymbol{B} \cdot \boldsymbol{\rho}) / \lambda=$ $\left(B_{x} a+B_{y} b\right) / \lambda$ is the phase difference of the two components in units of wavelength, $\beta$ is the introduced bandpass coefficient, and $f$ is the corresponding bandwidth-smearing coefficient, which is also $1 \sigma$ of the envelope function of the interferogram. The exact value of $f$ depends on the bandpass shape and windowing function. For example, for a "top-hat" bandpass approximation, $f \simeq$ 4.0; for a Gaussian bandpass approximation with FWHM $=\Delta \lambda$, $f \simeq 2$.6. We applied equation (2) to our squared-visibility model, with $f$ being a free parameter. The new best fit is significantly improved $\left(\chi_{\nu}^{2} \sim 1.3\right)$ compared to the preliminary result $\left(\chi_{\nu}^{2} \sim 1.9\right)$, giving $f$ a value of 3.4 , which is consistent with the fact that the bandpass of IOTA is between a top-hat and a Gaussian function. Figure 1 shows the best-fit squared-visibility models before and after applying the bandwidth-smearing correction. The data are plotted versus interferometric delay $\boldsymbol{B} \cdot \boldsymbol{\rho}$ (i.e., projected baseline times angular separation of the binary) in units of wavelength. The corresponding normalized residuals (i.e., normalized by their errors)

\footnotetext{
${ }^{8}$ This is equivalent to integrating the squared visibility over the full wavelength range to get the broadband value.
}

are shown in the right panels for the three baselines. As can be seen, the visibility amplitudes around \pm 1.0 and \pm 1.5 wavelengths in baseline $\mathrm{AB}$ (top) are reduced by a large amount from the original sinusoidal $V^{2}$ model because of the bandwidth-smearing effect, and the applied correction improved the fit significantly. Baselines $\mathrm{AC}$ and $\mathrm{BC}$ are shorter than baseline $\mathrm{AB}$ and therefore provide measurements with delay differences $<1$ wavelength and suffer less amplitude reduction than baseline AB.

A group of data around 1 wavelength in baseline AC from two different observations (2003 February 17 and 2004 April) have large normalized residuals $(>5)$ even after removing all known calibration errors. The orbital phases of these two epochs are $\sim 0.1$ and $\sim 0.20-0.25$, respectively. Inspection of these data revealed unusually high variations in the system visibilities on this baseline, indicating the poor fit at these epochs is likely due to calibration problems rather than errors in our determined orbital parameters.

\subsection{Bandwidth-Smearing Effect of Closure Phase and OPD Fluctuations}

Our preliminary best fit on closure phases also showed large residuals, leading to even larger $\chi_{\nu}^{2}(\sim 3)$ than that of the squared visibilities. This can also be the result of the bias induced by bandwidth smearing. However, unlike the case for the visibilities, this bias in the closure phases does not have a particularly simple analytical expression. One can only simulate this bias numerically, making it more difficult to look into the influence of bandwidth smearing. In our approach, we simulate the observational data of $\lambda$ Vir by generating three interferograms for the three IOTA baselines at different epochs. The different piezo scan modes are also taken into account. We then put the three interferograms into the IOTA data reduction pipeline ( $(2.2)$ to reproduce the "measured" closure phases as in real observations. We adopted the same bandpass function and bandwidth-smearing coefficient from the visibility modeling ( $\S 3.1$ ). By varying the width of the interferogram envelope function, we simulated the closure phases for both the monochromatic and the polychromatic cases. 

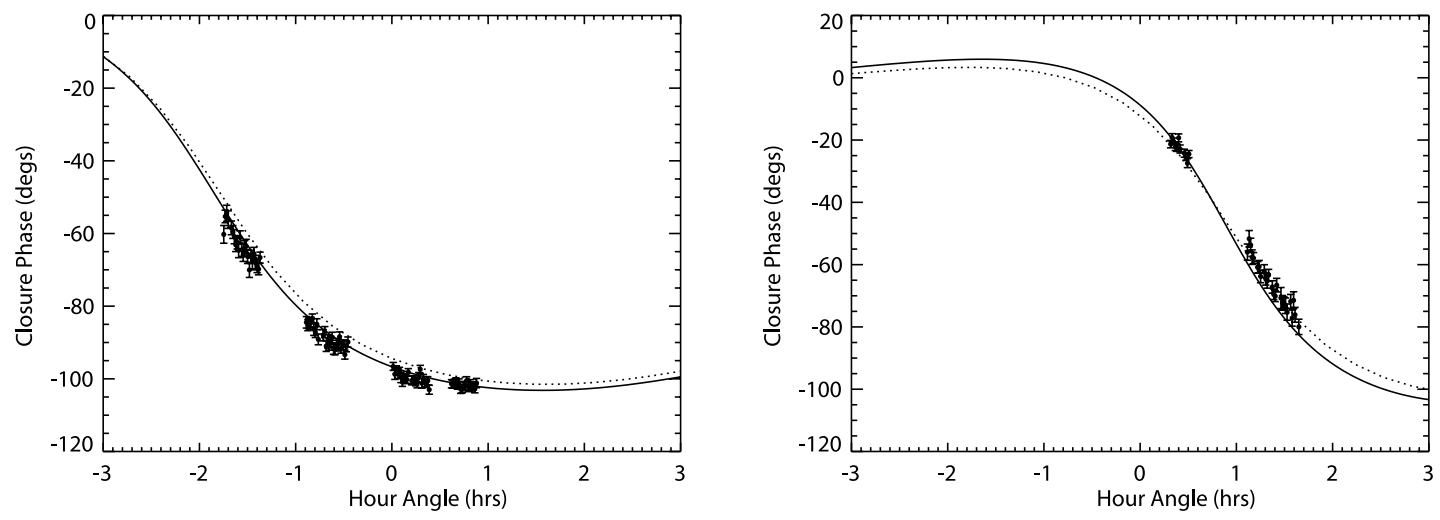

FIG. 2.-Preliminary closure-phase model vs. hour angle. Two typical dates of data with different scan modes (left: 2003 March 24, scan mode 1; right: 2005 June 16, scan mode 2) are selected to represent the whole data set. The solid lines show the original closure-phase model, while the dotted lines show the model with bandwidth smearing taken into account. The difference between the two models is about $5^{\circ}$ in both panels. Closure-phase data are indicated as filled dots with $1 \sigma$ measurement errors.

Figure 2 shows the bandwidth-smearing-corrected (dotted lines) and the original uncorrected (solid lines) closure-phase models for two typical observations (2003 March 24 and 2005 June 16). These two observations represent two different scanning modes: mode 1 for 2003 March 24 (left) and mode 2 for 2005 June 16 (right). Figure 2 indicates that bandwidth smearing can change the closure phases by $\sim 5^{\circ}$ at these two epochs. Although the fit is improved in the right panel by the simulated bandwidth-smearing model, the one in the left is worse than the original fit. In fact, the original model deviates from the measured closure phases by up to $10^{\circ}$ in the whole data set, and the simulated bandwidth smearing cannot reduce these deviations significantly, implying other biases may exist in the closure-phase measurements.

Another source of error in the closure phases stems from the offsets of the fringe phases due to extra OPDs induced by the atmospheric piston fluctuations. Further investigations (see the Appendix) suggest that this effect does dominate the errors of our closure-phase measurements. To reduce the influence of this effect on our fits, we estimate the errors of the closure phases based on their uncertainties obtained from the simulations of closurephase fluctuations caused by extra OPDs. The details of the simulation and the corresponding closure-phase behaviors are discussed in the Appendix. Figure 3 shows the best-fit closurephase model for the two typical observations, overplotted with the observed data and the estimated errors. The errors in the first epoch (left) are much smaller than those in the second epoch due to their differing scan modes. The best fit leads to a $\chi_{\nu}^{2}$ of 1.2 with
476 degrees of freedom for the closure phases (previously $\chi_{\nu}^{2} \sim$ 3 ), which is significantly reduced as a result of the reliable error estimation.

\subsection{The Final Orbit}

With the bandwidth-smearing effects addressed as described above, we proceed in this section with a simultaneous Keplerian orbital fit to the radial velocities, the squared visibilities, and the closure phases for $\lambda$ Vir. This allows us to determine the full set of orbital elements, for which the closure phases remove the ambiguity in the position angle of the ascending node $(\Omega)$ that is usually inherent in the visibility measurements. The inclination angle $i$ is determined from the interferometric data, and consequently the masses $M_{1}$ and $M_{2}$ can be found from the spectroscopic values of $M_{1} \sin ^{3} i$ and $M_{2} \sin ^{3} i$. Since neither of the $\lambda$ Vir components are resolved by IOTA, we take the sizes of the two components into account by using a uniform stellar disk model (Boden 2000). The applied diameters, 0.40 mas for the primary and 0.30 mas for the secondary, are consistent with the values determined in $\S 5$. The overall $\chi^{2}$ of the measurements is minimized using standard nonlinear least-squares techniques, in our case, the Levenberg-Marquardt algorithm, and the errors of the best-fit parameters are estimated using the bootstrap method (Press et al. 1992)

The calibration error of the squared visibilities is obtained from the fitting procedures, leading to a value of $\Delta V^{2}=0.017$ that corresponds to a $1.7 \%$ error for an unresolved source $\left(V^{2}=1\right)$.
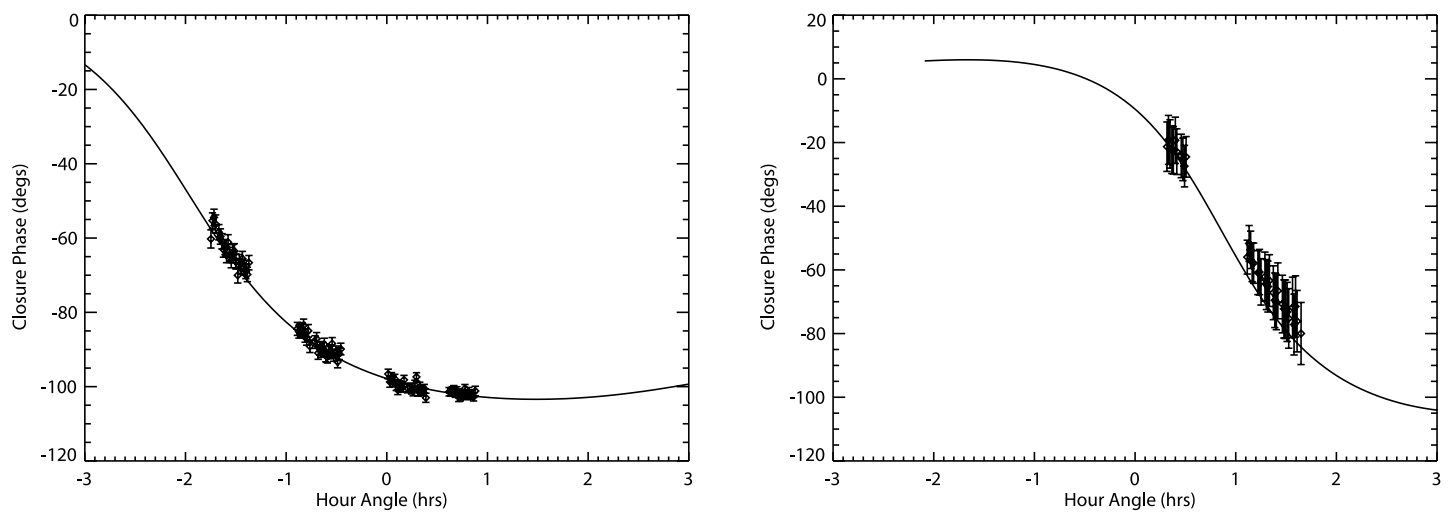

FIG. 3.-Closure-phase model and data with new estimated errors. The two panels have the same dates as in Fig. 2. The new $1 \sigma$ errors in the left panel are smaller than those in the right one due to smaller closure-phase fluctuations in scan mode 1. The good fit of the data within the errors suggests the robustness of our error estimation. 

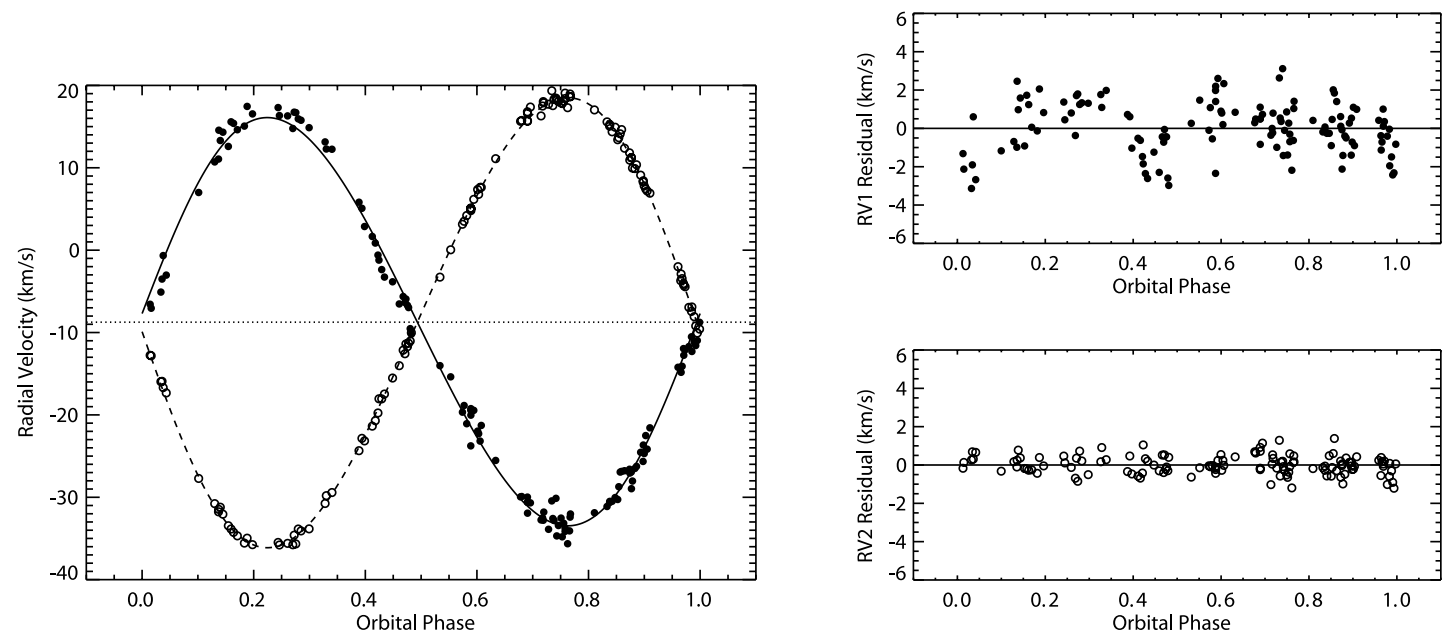

FIG. 4.-Best-fit radial velocity model vs. orbital phase. The data are shown with filled circles for the primary and open circles for the secondary. The best-fit radial velocity curves are also shown (solid line, primary; dashed line, secondary). The dotted line indicates the systemic velocity of the primary. Secondary velocities have been corrected for the offset described in the text. Velocity residuals are given in the right panels. The larger values for the primary are caused by the larger rotational broadening of its spectral lines and possibly by template mismatch due to the anomalous abundances (see text).

Closure-phase errors are determined in $\S 3.2$. The statistical weights (or errors) of the radial velocity data are established from the model-fitting procedures as well. In particular, we start with initial values and iterate the $\chi^{2}$ minimization for the primary and the secondary radial velocities until the estimated weights converge. The resulting error for the primary, $1.34 \mathrm{~km} \mathrm{~s}^{-1}$, is larger than that of the secondary, $0.50 \mathrm{~km} \mathrm{~s}^{-1}$, due to the fact that the primary is broad-lined and the secondary is sharp-lined. Figure 4 shows the radial velocity models plotted versus orbital phase. In the fit we allowed for a possible offset between the primary and secondary velocities that could originate from a template mismatch in the cross-correlations due to the metallic-lined nature of the stars (see $\S 2.1$ ). We found a small but statistically significant offset of $0.70 \pm 0.13 \mathrm{~km} \mathrm{~s}^{-1}$, which has been accounted for in plotting the secondary velocities. The corresponding best-fit residuals are given in the right panels. It is noticeable that the primary has much larger residuals than the secondary. We searched carefully for the presence of a third star that might be responsible for fluctuations in the orbit. However, neither the spectroscopic or interferometric data, nor the Hipparcos data and other available online catalogs (such as Two Micron All Sky Survey [2MASS]) indicate any such evidence. Keck aperture masking was also used on this source, and no wider companions $\left(\rho<0.5^{\prime \prime}\right)$ were observed at $2 \mu \mathrm{m}$ (J. D. Monnier 2005, private communication). These investigations indicate the absence of a third companion within the detection limits, and even if it exists, it would have negligible influence on the $\lambda$ Vir orbit. The temperature and luminosity of the primary star are typical of $\delta$ Scuti variables, which have pulsation periods usually less than 0.3 days. Our velocity sampling is not well suited to discover periodicities as short as this. However, it is unlikely that oscillations of this kind contribute significantly to the velocity residuals we see in Figure 4. Instead, the pattern suggests a much longer period variation (quite apparent in the figure, at least between phase 0.0 and 0.5 ). Indeed, a periodogram analysis of the residuals shows significant power at a period very close to half the orbital period. We believe the source of these residuals is template mismatch, caused by the anomalous abundances of the stars. The primary is more vulnerable to these effects due to its broader lines. The dependence with phase comes from the unavoidable fact that different spectral lines shift in and out of our spectral window as the stars orbit each other.
The preliminary orbital parameters are shown in the third column of Table 3 . As can be seen in the table, the $\chi_{\nu}^{2}$ of the squared visibilities and closure phases are still larger than unity. In fact, these large $\chi_{\nu}^{2}$ values are due to the systematic bias in the closure phases caused by the bandwidth-smearing effect mentioned previously and also in the Appendix, which tends to change the flux ratio and cannot be eliminated by the new estimated errors. In order to reduce this bias and other uncertainties in the closure phases, we conservatively give small weight to the closure phases in the fit such that the orbital parameters primarily come from the squared visibilities and the radial velocities. The weight for the closure phases is determined iteratively in the fit until the deweighting of closure phases does not change the flux ratio any more. Figure 5 depicts the best-fit visual orbit of $\lambda$ Vir, and the final best-fit parameters are listed in the fourth column of Table 3 . The deweighting of the closure phases also improved the $\chi_{\nu}^{2}$ of the visibilities, as well as the overall fit. The value of the flux ratio increased a significant amount due to the elimination of the closure-phase bias. For reference, we also list the parameters from Stickland (1975) in the table. Due to the measurement uncertainties of Stickland (1975) and the near-equal masses of the two components, the primary and secondary components were reversed, resulting in a $180^{\circ}$ difference in $\omega$ compared to our value. We have corrected this in Table 3.

\section{PHYSICAL PARAMETERS}

The combination of the astrometric and spectroscopic information provided by our orbital solution yields precise estimates of the absolute masses of the components of $\lambda \mathrm{Vir}$, with relative errors of only $0.7 \%$ for the primary and $1.5 \%$ for the secondary. These are listed in Table 4 along with other physical parameters described below. We use these in $\S 5$ to compare with recent stellar evolution models and assess the evolutionary state of the system.

The system bolometric flux and luminosities are determined through spectral energy distribution (SED) modeling. We constructed two-component SED models using both Kurucz (Kurucz et al. 1974) and Pickles ${ }^{9}$ model templates and applied them to a substantial amount of archive photometric measurements in the

${ }^{9}$ Vizier Online Data Catalog, J/PASP/110/863 (A. J. Pickles, 1998). 
TABLE 3

Orbital and Binary Parameters of $\lambda$ V Ir

\begin{tabular}{|c|c|c|c|}
\hline $\begin{array}{l}\text { Parameter } \\
\text { (1) }\end{array}$ & $\begin{array}{c}\text { Stickland }(1975)^{\mathrm{a}} \\
\text { (2) }\end{array}$ & $\begin{array}{l}\text { Preliminary } \mathrm{Fit}^{\mathrm{b}} \\
\text { (3) }\end{array}$ & $\begin{array}{l}\text { Best Fit }{ }^{\mathrm{c}} \\
\text { (4) }\end{array}$ \\
\hline$H$-band flux ratio $\ldots \ldots \ldots \ldots \ldots \ldots$ & & $0.5749 \pm 0.0021$ & $0.6055 \pm 0.0056$ \\
\hline 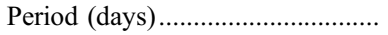 & $206.64 \pm 0.05$ & $206.7323 \pm 0.0061$ & $206.7321 \pm 0.0040$ \\
\hline$T_{0}(\mathrm{MJD}) \ldots$ & $40,253.1 \pm 15.5$ & $53,070.28 \pm 0.50$ & $53,070.30 \pm 0.32$ \\
\hline 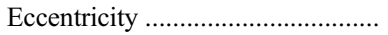 & $0.079 \pm 0.021$ & $0.0603 \pm 0.0031$ & $0.0610 \pm 0.0036$ \\
\hline$\omega(\operatorname{deg})$ & $273.3 \pm 26.8$ & $272.10 \pm 0.71$ & $272.28 \pm 0.46$ \\
\hline$\Omega(\operatorname{deg})$ & & $196.57 \pm 0.16$ & $196.40 \pm 0.22$ \\
\hline$i(\mathrm{deg})$ & & $109.97 \pm 0.15$ & $109.86 \pm 0.24$ \\
\hline$a$ (mas) & & $19.768 \pm 0.072$ & $19.759 \pm 0.079$ \\
\hline$K_{1}\left(\mathrm{~km} \mathrm{~s}^{-1}\right) \ldots \ldots$ & $29.51 \pm 0.89$ & $24.78 \pm 0.17$ & $24.78 \pm 0.17$ \\
\hline$K_{2}\left(\mathrm{~km} \mathrm{~s}^{-1}\right) \ldots \ldots$ & $24.85 \pm 0.65$ & $27.308 \pm 0.067$ & $27.308 \pm 0.067$ \\
\hline$\Delta \mathrm{RV}\left(\mathrm{km} \mathrm{s}^{-1}\right) \ldots \ldots \ldots \ldots \ldots \ldots$ & & $-0.69 \pm 0.13$ & $-0.70 \pm 0.13$ \\
\hline$\gamma\left(\mathrm{km} \mathrm{s}^{-1}\right)$ & $-6.40 \pm 0.41$ & $-8.053 \pm 0.045$ & $-8.053 \pm 0.045$ \\
\hline$f$ coefficient $^{\mathrm{d}} \ldots \ldots \ldots \ldots$ & & $3.47 \pm 0.18$ & $3.08 \pm 0.14$ \\
\hline 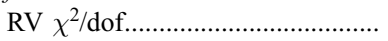 & & 1.02 & 1.02 \\
\hline$V^{2} \chi^{2} /$ dof $\ldots \ldots \ldots \ldots$ & & 1.40 & 1.03 \\
\hline $\mathrm{CP} \chi^{2} /$ dof & & 1.21 & 0.12 \\
\hline Total $\chi^{2} /$ dof............................... & & 1.21 & 0.89 \\
\hline
\end{tabular}

a Due to measurement uncertainties in Stickland's work the primary and secondary components are reversed, resulting in a value of $\omega$ that differs from ours by $180^{\circ}$. This has been corrected in the table.

${ }^{b}$ Preliminary orbit fit using bandwidth-smearing-corrected $V^{2}$ model and reestimated closure-phase errors (see $\S 3.3$ ).

${ }^{c}$ Closure phases are deweighted in the best fit to eliminate biases and uncertainties, especially those in the flux ratio.

d The introduced bandwidth-smearing coefficient (see $\S 3.1$ ).

Johnson, Strömgren, Geneva, and 2MASS systems, as well as spectrophotometric measurements from Breger (1976), Burnashev (1985), and Glushneva et al. ${ }^{10}$ However, the Burnashev (1985) and Glushneva et al. spectrophotometry are not consistent with photometry at wavelengths longer than $420 \mathrm{~nm}$, and the Burnashev (1985) data also have bad normalizations that do not agree with

${ }^{10}$ Vizier Online Data Catalog, 3208 (I. N. Glushneva et al., 1998).

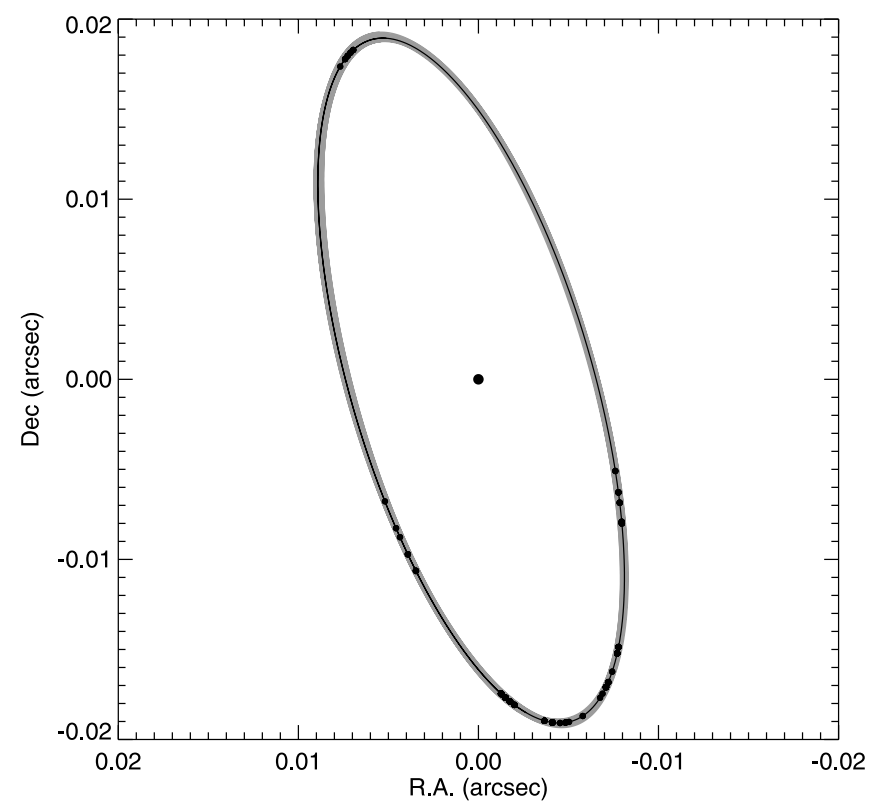

FIG. 5.-Best-fit visual orbit of $\lambda$ Vir. The primary is shown by the central dot. The solid line indicates the best-fit orbit, and the overplotted filled dots show the epochs of interferometric observations. The shaded area around the orbit indicates the $1 \sigma$ uncertainties of the orbit. [See the electronic edition of the Journal for a color version of this figure.] other data. Therefore, we only take the spectrophotometry of Breger (1976) into account in our fitting. The component light ratios determined from spectroscopy and interferometry in $\S 2$ are also used to constrain the models. After extensive tests of model templates, we found that the Pickles templates are not appropriate for $\lambda$ Vir because of its metallic-lined nature. The Kurucz model with $[m / H]=+0.5$ best fits the data. Figure 6 depicts the resulting best-fit Kurucz model, as well as the corresponding SEDs for both the primary and the secondary, overplotted with the input flux measurements and the model net flux for corresponding bandpasses. The best-fit model calls for two A1 V stars with no need of extinction correction. The resulting system bolometric flux is $(3.794 \pm 0.014) \times 10^{-7} \mathrm{ergs} \mathrm{cm}^{-2} \mathrm{~s}^{-1}$. With the distance determined below, the luminosities for the primary and the secondary are $20.84 \pm 0.25$ and $12.58 \pm 0.16 L_{\odot}$, respectively.

The effective temperature estimates in $\S 2.1$ are strongly correlated with the metallicity adopted for $\lambda \mathrm{Vir}$, in the sense that higher metallicities lead to higher temperatures. Consequently, because the composition in the surface layers of $\lambda$ Vir is enhanced compared to normal A stars, our temperatures are likely to be overestimated. We therefore made use of extensive photometric measurements available for the object in the Johnson, Strömgren, and Geneva systems (Mermilliod et al. 1997), as well as 2MASS, to derive the mean effective temperature based on a large number of color/temperature calibrations (Popper 1980; Moon \& Dworetsky 1985; Blackwell et al. 1990; Gray 1992; Napiwotzki et al. 1993; Balona 1994; Smalley \& Dworetsky 1995; Kunzli et al. 1997; Cox 2000). In addition we made an estimate by the infrared flux method (Blackwell et al. 1990) based on the bolometric flux determined from the SED, the flux from the 2MASS $K_{s}$ band, and the corresponding integrated flux from the Kurucz model. The various estimates are in good agreement, showing a scatter of about $120 \mathrm{~K}$ and yielding an average of $T_{\mathrm{eff}}=8280 \pm$ $200 \mathrm{~K}$, where the uncertainty is a conservative estimate to account also for the possibility of systematic errors in the calibrations. To 
TABLE 4

Physical Parameters of $\lambda$ Vir

\begin{tabular}{|c|c|c|c|}
\hline Physical Parameter & Value & Primary Component & Secondary Component \\
\hline Mass $\left(M_{\odot}\right)^{\mathrm{a}} \ldots \ldots \ldots$ & & $1.897 \pm 0.016$ & $1.721 \pm 0.023$ \\
\hline$\pi_{\text {orb }}(\mathrm{mas})^{\mathrm{a}}$ & $18.81 \pm 0.10$ & & \\
\hline$\pi_{\mathrm{HIP}}^{\prime}(\mathrm{mas})^{\mathrm{b}} \ldots \ldots \ldots \ldots \ldots$ & $18.55 \pm 0.84$ & & \\
\hline System distance $(\mathrm{pc})^{\mathrm{a}}$ & $53.16 \pm 0.29$ & & \\
\hline Semimajor axis $(\mathrm{AU})^{\mathrm{c}}$ & $1.0504 \pm 0.0071$ & & \\
\hline Visible light ratio & $0.56 \pm 0.10$ & & \\
\hline$H$-band flux ratio ${ }^{\mathrm{c}}$ & $0.6055 \pm 0.0056$ & & \\
\hline$V$ magnitude (mag) & & $5.003 \pm 0.070$ & $5.63 \pm 0.12$ \\
\hline Bolometric flux $\left(10^{-7} \mathrm{ergs} \mathrm{cm}^{-2} \mathrm{~s}^{-1}\right) \ldots \ldots \ldots \ldots \ldots \ldots \ldots$ & & $2.366 \pm 0.010$ & $1.428 \pm 0.089$ \\
\hline Total bolometric flux $\left(10^{-7} \mathrm{ergs}^{-2} \mathrm{~cm}^{-1}\right) \ldots \ldots \ldots \ldots \ldots$ & $3.794 \pm 0.014$ & & \\
\hline Luminosity $\left(L_{\odot}\right)$ & & $20.84 \pm 0.25$ & $12.58 \pm 0.16$ \\
\hline$T_{\text {eff }}(\mathrm{K})$ & & $8280 \pm 200$ & $8280 \pm 200$ \\
\hline$v \sin i\left(\mathrm{~km} \mathrm{~s}^{-1}\right)$ & & $36 \pm 1$ & $10 \pm 2$ \\
\hline
\end{tabular}

${ }^{\text {a }}$ Parameters that are determined directly from the best-fit orbital parameters.

${ }^{\mathrm{b}}$ Revised Hipparcos parallax accounting for orbital motion.

${ }^{c}$ From Table 3.

the extent that the abundance enhancements of the two stars are similar (which appears to be the case, as reported by Stickland 1975), our spectroscopic analysis in $\S 2.1$ indicates no significant difference in temperature between the stars. Reddening estimates based on Geneva and Strömgren photometry give negligible values using calibrations by Crawford (1979) and Kunzli et al. (1997) consistent with the relatively close distance to the object.

The orbital parallax of the system is $\pi_{\mathrm{orb}}=18.81 \pm 0.10 \mathrm{mas}$, corresponding to a distance of $53.16 \pm 0.29 \mathrm{pc}$. The trigonometric parallax listed in the Hipparcos catalog is $\pi_{\mathrm{HIP}}=17.47 \pm$ 0.94 mas, which is slightly lower than ours (a $1.4 \sigma$, or $7 \%$ effect), most likely because it does not account for the perturbation from the orbital motion. The original Hipparcos observations are available in the form of "abscissa residuals," which are the one-

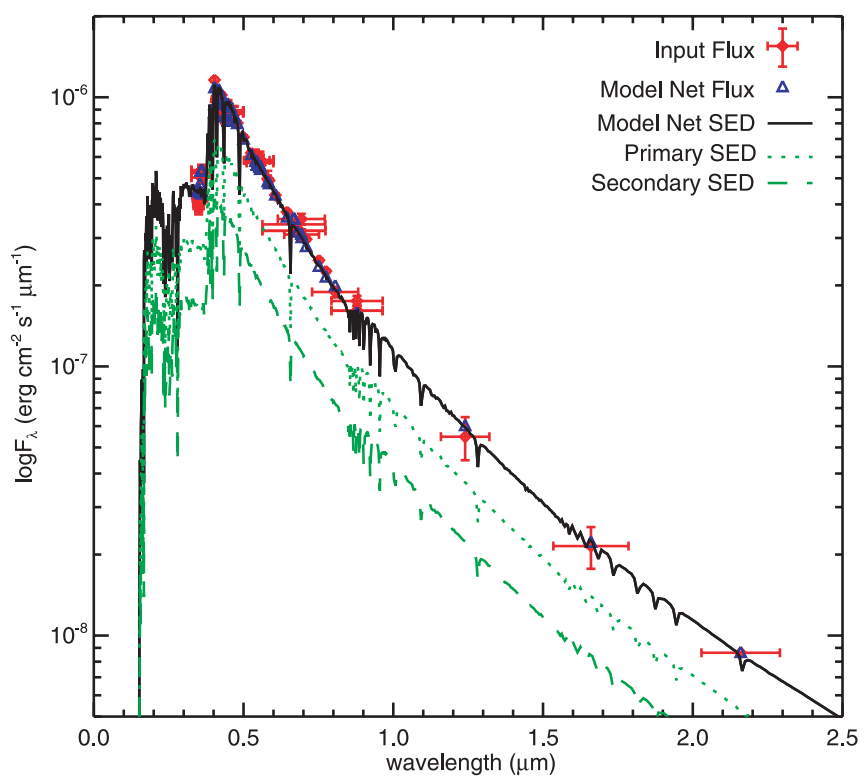

FIG. 6.-SED models of $\lambda$ Vir. The net SED model is shown by the solid line, overplotted with input flux and the bandpass-integrated model flux. The bandpass of input fluxes are shown by the horizontal error bars. The SED for the primary is shown by the dotted line and the secondary by the dashed line. The models correspond to two A1 V stars. dimensional residuals (along the scan direction of the satellite) from the usual five-parameter solutions yielding the position, proper motion, and parallax as reported in the Hipparcos and Tycho Catalogues. ${ }^{11}$ We have rereduced these measurements by expanding the model to account for the orbital motion constrained using our own solution, and we have solved for the semimajor axis of the photocenter $\left(a_{\text {phot }}\right)$, as well as corrections to the position and proper motion of the barycenter and a correction to the parallax. The formalism for this solution follows closely that described by van Leeuwen \& Evans (1998) and Pourbaix \& Jorissen (2000), and a recent example of a similar application is given by Torres (2006). The revised Hipparcos parallax we obtain is $\pi_{\mathrm{HIP}}^{\prime}=$ $18.55 \pm 0.84$ mas, which is now in much better agreement with $\pi_{\text {orb }}$ (within $0.3 \sigma$ ). The motion of the center of light of the binary is clearly detected by Hipparcos, albeit with much lower precision than the relative semimajor axis, and amounts to $a_{\text {phot }}=3.84 \pm 0.63$ mas. $^{12}$

This value, along with the relative semimajor axis and the mass ratio, allows us to obtain an independent estimate of the light ratio in the Hipparcos passband $\left(H_{p}\right)$, which is $\ell_{2} / \ell_{1}=0.39 \pm 0.06$. This is significantly lower than the spectroscopic and interferometric value in $\S 2$. There is no evidence from either the spectroscopy or the interferometry of any photometric variability in $\lambda$ Vir that might explain the difference, in agreement with the small scatter observed in the brightness measurements from Hipparcos $\left(\sigma_{H_{p}}=0.006 \mathrm{mag}\right) .{ }^{13}$ The small amplitude of the photocentric motion compared to the median error of an individual abscissa residual (2.3 mas) may be cause for some concern about possible systematics in the Hipparcos light ratio, although we have no independent evidence for such an effect. On the other hand, in view of the metallic-lined nature of the stars we cannot entirely rule out the possibility of a bias in the spectroscopic value of $\ell_{2} / \ell_{1}$ of a similar nature as the effect in the temperatures mentioned above. However, the brightness ratio is a differential measurement, and therefore, we would not expect the effect to be large.

\footnotetext{
11 Vizier Online Data Catalog, 1239 (ESA, 1997).

12 For completeness we list here the remaining parameters adjusted in this fit: $\Delta \alpha \cos \delta=-0.19 \pm 0.77$ mas, $\Delta \delta=+0.03 \pm 0.47$ mas, $\Delta \mu_{\alpha} \cos \delta=+1.48 \pm$ $0.81 \mathrm{mas} \mathrm{yr}^{-1}$, and $\Delta \mu_{\delta}=+0.47 \pm 0.58 \mathrm{mas} \mathrm{yr}^{-1}$. These should be added with their sign to the catalog values of the position and proper motion to yield the revised values.

13 Vizier Online Data Catalog, 1239 (ESA, 1997).
} 
Since both light-ratio estimates are close to the visual band and the stars are of similar temperature, for the purpose of the modeling in $\S 5$ we have chosen as a compromise to adopt the weighted average of the spectroscopic and Hipparcos values. That average is $\ell_{2} / \ell_{1}=0.56 \pm 0.10$. The larger uncertainty accounts for the individual weights, as well as the difference in the values themselves.

The absolute visual magnitudes of the components follow from this value, along with the orbital parallax and the apparent system magnitude of $V=4.52 \pm 0.01$ (Mermilliod et al. 1997), and are included in Table 4. Although we have derived a very precise flux ratio in the $H$ band from the interferometric observations, a total $H$-band magnitude for the system is unavailable (the star is bright enough that it saturated the 2MASS detector), and so the individual magnitudes cannot be computed directly.

\section{COMPARISON WITH STELLAR EVOLUTION MODELS}

The accurately measured masses, absolute visual magnitudes, and effective temperatures of the components of $\lambda$ Vir, as well as the flux ratio in the $H$ band, allow a comparison with current models of stellar evolution. For their ease of use we have chosen here the Yonsei-Yale series of calculations by Yi et al. (2001) and Demarque et al. (2004). The color/temperature transformations and bolometric corrections adopted are those of Lejeune et al. (1998), and the passband of the $H$ filter in those calculations is sufficiently close to that used at IOTA for our purposes. Unfortunately the actual bulk composition of $\lambda$ Vir is difficult or impossible to determine observationally because of the metallic-lined nature of both stars. Therefore, we have explored a range of interior metallicities in the models to identify the values that are consistent with the observations.

Initially we considered only the masses, absolute magnitudes, and effective temperatures of the two stars as constraints. By interpolation we computed a fine grid of isochrones for a large number of age and metallicity combinations, and compared each model with the six measurements under the assumption that the stars are coeval and have the same interior composition. The result is shown in Figure 7, where each filled circle represents an age/metallicity combination that agrees with the observations for both stars within the errors. The best agreement occurs near the center of the region (larger filled circles), at a metallicity near $Z=0.01$ and an age of about 900 Myr. Next we added the constraint on the flux ratio in $H$, requiring that in addition to matching the masses, magnitudes, and temperatures, the models reproduce the observed magnitude difference in $H$. The combinations that also satisfy this last constraint cover a smaller area of the diagram and are indicated with open circles in Figure 7. The best overall match is achieved for a metallicity of $Z=0.0097$ (corresponding to $[\mathrm{Fe} / \mathrm{H}]=-0.29$, assuming no enhancement of the alpha elements) and an age $t$ of $935 \mathrm{Myr}$, at which the models agree with all observables well within the errors (typically to better than $0.4 \sigma$ ).

The comparison of the masses, absolute magnitudes, and temperatures with the models is shown graphically in Figure 8. The solid lines in the top panel represent evolutionary tracks computed for the exact masses we measured for each star, and the dotted lines indicate the uncertainty associated with the mass errors $( \pm 1 \sigma)$. The 935 Myr isochrone is shown as a dashed line and indicates that the components of $\lambda$ Vir are indeed consistent with having the same age, as expected. Figure $8 b$ shows the best-fit model isochrone and the observations in the mass-luminosity diagram. The constraint on the flux ratio is illustrated in Figure 9, where we have chosen to represent the predicted magnitude difference

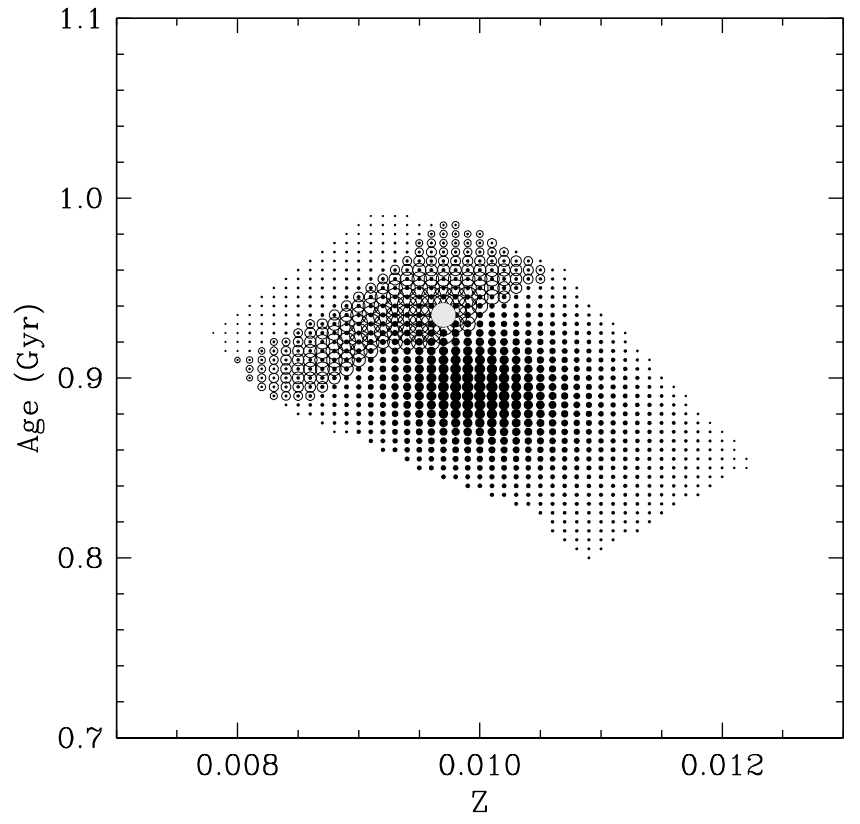

FIG. 7.-Determination of the age and metallicity of $\lambda$ Vir by comparison with stellar evolution models by Yi et al. (2001) and Demarque et al. (2004). Filled circles show all age/metallicity combinations that yield an isochrone matching the measured values of the mass, effective temperature, and absolute visual magnitude of both stars (assumed to be coeval) within the observational errors (Table 4). Larger filled circles indicate a better match. Open circles indicate age/metallicity combinations that also satisfy the measured flux ratio in the $H$ band, within its uncertainty. The size of the open circles is again proportional to the goodness of fit. The best overall match to the observations is indicated with the large gray circle and corresponds to $Z=0.0097$ (or $[\mathrm{Fe} / \mathrm{H}]=-0.29$ ) and an age of $935 \mathrm{Myr}$.

from the model (solid line) as a function of the primary mass, with the secondary mass being determined at each point along the curve from the measured mass ratio $\left(q \equiv M_{2} / M_{1}\right)$. The dotted lines represent the uncertainty in the location of this curve $( \pm 1 \sigma)$ resulting from the error in $q$. The measurement is in good agreement with the predictions.

The estimated radii of the stars from the best-fitting model are $R_{1}=2.35 R_{\odot}$ and $R_{2}=1.84 R_{\odot}$ for the primary and secondary, respectively, and the corresponding angular diameters at the distance of $\lambda$ Vir are $\phi_{1}=0.41$ mas and $\phi_{2}=0.32$ mas. These are not far from the values adopted for the orbital solution described in $\S 3.3$. The surface gravities are $\log g_{1}=3.97$ and $\log g_{2}=$ 4.14, which are close to the value of $\log g=4.0$ adopted for both components in $\S 2.1$.

\section{COMPARISON WITH TIDAL THEORY}

The measures of the absolute dimensions, as well as the projected rotational velocities $v \sin i$ of the components of $\lambda$ Vir, allow us to test various aspects of tidal evolution theory. Tidal forces in binaries tend to synchronize the rotation of each star to the mean orbital motion, to align the spin axes of the stars with the axis of the orbit, and to circularize the orbit.

In general the timescales for these processes are very different (see, e.g., Hut 1981). Alignment and synchronization typically proceed much more quickly than circularization, often by an order of magnitude or more when the angular momentum of the orbit is larger than the rotational angular momentum. Tidal forces are highly sensitive to the dimensions and structure of the stars. Both components of $\lambda$ Vir started their main-sequence lives with 

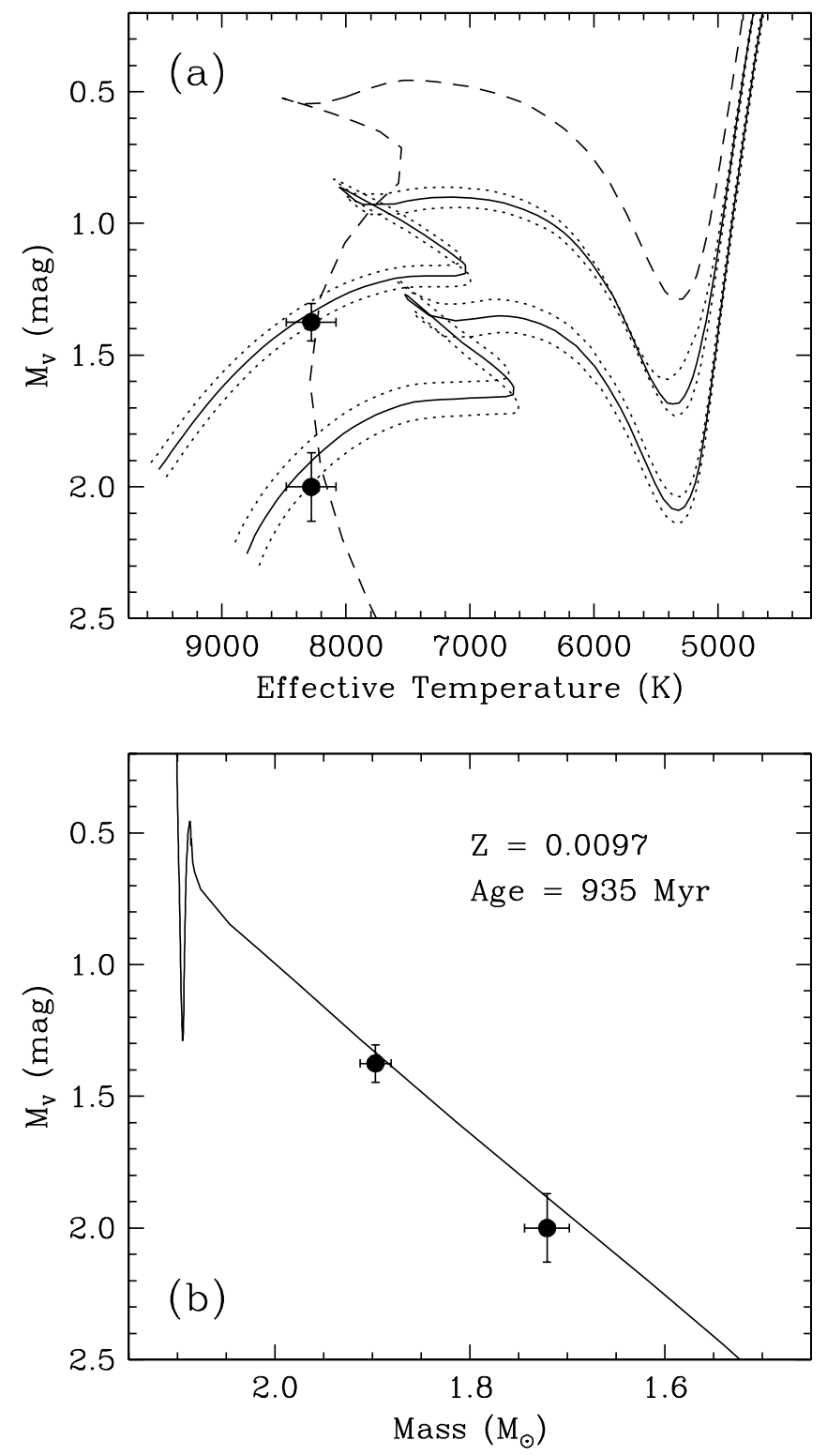

FIG. 8.-Comparison between the measurements for $\lambda$ Vir and the bestmatching stellar evolution models by Yi et al. (2001) and Demarque et al. (2004) for a metallicity of $Z=0.0097$ (or $[\mathrm{Fe} / \mathrm{H}]=-0.29$ ) and an age of $935 \mathrm{Myr}$. (a) Evolutionary tracks in the absolute magnitude/effective temperature diagram for the exact masses measured for each star (solid lines). The uncertainty in the location of the tracks stemming from the mass errors $( \pm 1 \sigma)$ is represented with the dotted lines. The $935 \mathrm{Myr}$ isochrone is shown by the dashed line. (b) Best-fitting isochrone in the mass-luminosity diagram.

convective cores and radiative envelopes, but in later evolutionary stages their envelopes will become convective. Therefore, it is necessary to consider two different mechanisms of tidal braking appropriate for each stage, which are referred to as radiative damping and turbulent dissipation, respectively (Zahn 1977, 1989). The timescales for synchronization and circularization for the case of stars with convective envelopes are given by

$$
\begin{gathered}
\tau_{\text {sync }}=3.95 \times 10^{2} \beta^{2} M^{7 / 3} \frac{(1+q)^{2}}{q^{2}} L^{-1 / 3} \lambda_{2}^{-1} \frac{P^{4}}{R^{16 / 3}} \\
\tau_{\text {circ }}=1.99 \times 10^{3} M^{3} \frac{(1+q)^{5 / 3}}{q} L^{-1 / 3} \lambda_{2}^{-1} \frac{P^{16 / 3}}{R^{22 / 3}}
\end{gathered}
$$

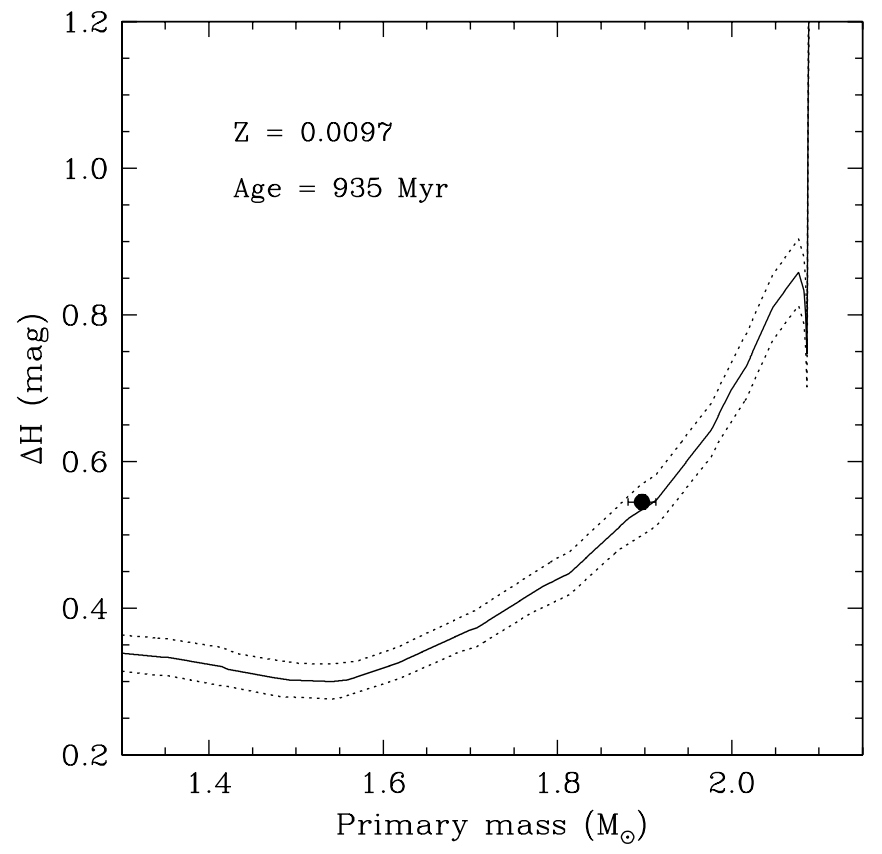

FIG. 9.- Predicted magnitude difference in the $H$ band from the best-fitting model isochrone for $\lambda$ Vir, compared with our accurate measurement from IOTA (vertical error bar smaller than the size of the point). The solid line is the prediction for the exact mass ratio $q$ we measure. At each point along this line the secondary mass is computed from the primary mass and $q$, and the magnitude difference is read off from the isochrone. The dotted lines represent the uncertainty in the prediction resulting from the error in $q$.

while for stars with convective cores and radiative envelopes the timescales are

$$
\begin{aligned}
& \tau_{\text {sync }}=2.03 \beta^{2} M^{7 / 3} \frac{(1+q)^{2}}{q^{2}} E_{2}^{-1} \frac{P^{17 / 3}}{R^{7}} \\
& \tau_{\text {circ }}=1.71 \times 10^{1} M^{3} \frac{(1+q)^{5 / 3}}{q} E_{2}^{-1} \frac{P^{7}}{R^{9}} .
\end{aligned}
$$

In the above expressions the timescales are given in years, $q$ represents the mass ratio, and $M, R$, and $L$ are the mass, radius, and luminosity in solar units, respectively. The period $P$ is given in days. The symbol $\lambda_{2}$ represents the tidal coefficient (see Zahn 1989), $\beta$ is the fractional radius of gyration, and the coefficient $E_{2}$ is related to the dynamical tidal contribution to the total perturbed potential (see Claret \& Cunha 1997, eq. [6] and following). In order to consider the contribution of both components to the circularization we use the equivalent timescale

$$
-\frac{1}{e} \frac{d e}{d t}=\frac{1}{\tau_{\text {circ }, 1}}+\frac{1}{\tau_{\text {circ }, 2}}
$$

where subscripts 1 and 2 refer to the primary and the secondary components, respectively. The differential equations that govern the evolution of the eccentricity and axial rotation were integrated along evolutionary tracks for each star until the relative variations reached $0.05 \%$ of their initial values. The integrations were carried out using the fourth-order Runge-Kutta method.

We find that the time of circularization of the orbit is predicted to be $t_{\text {circ }}=1.245 \mathrm{Gyr}$, which is larger than the present age of the system. Thus, the nonzero eccentricity we measure for the orbit of $\lambda \operatorname{Vir}(e=0.0610 \pm 0.0036)$ is consistent with theory. The primary star is expected to become synchronized with the mean orbital motion at a slightly earlier time $t_{\text {sync }, 1}=1.239$ Gyr. Once 

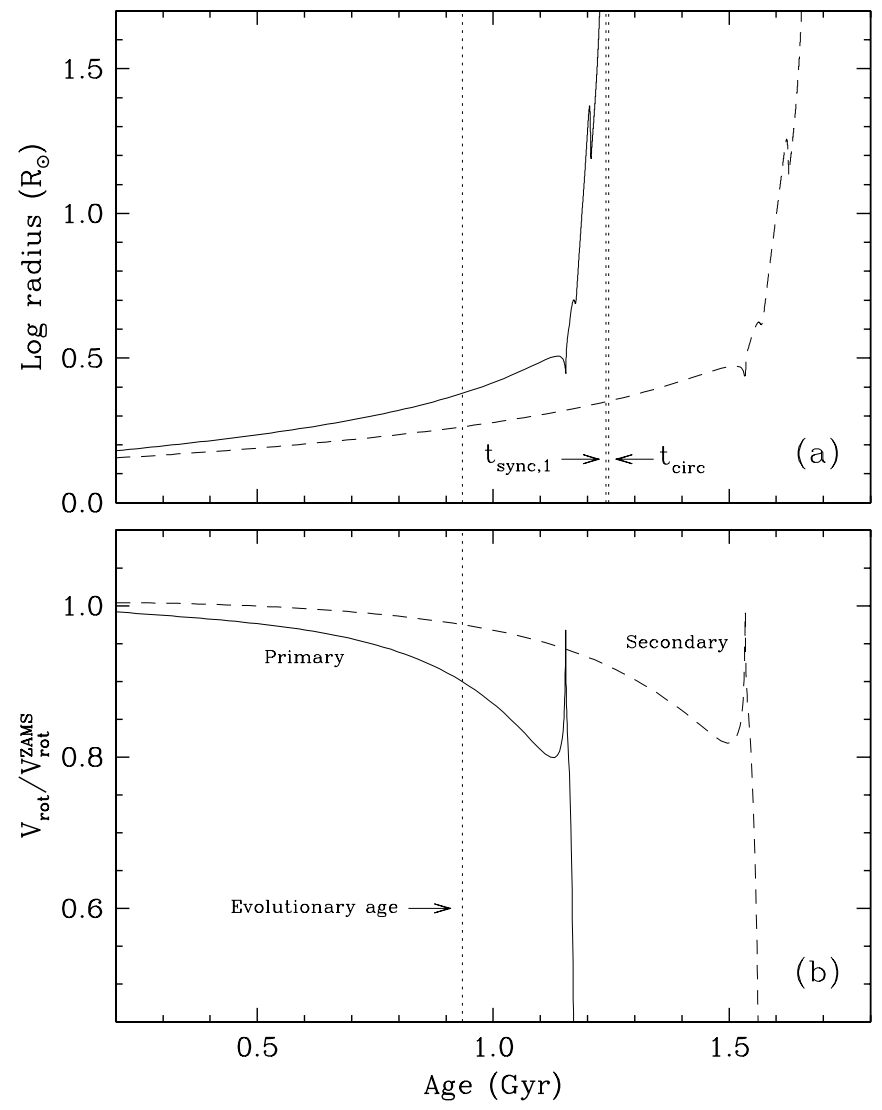

Fig. 10.-Tidal evolution of $\lambda$ Vir. (a) Expected change in radius as a function of time based on the models by Claret (2004). The present evolutionary age of the binary is indicated, along with the predicted times of synchronization of the primary and of circularization of the orbit due to tidal forces. (b) Evolution of the rotational velocity of each component relative to the initial rotation on arrival on the ZAMS, due solely to the change in the moment of inertia with time.

again this agrees with theoretical expectations, since the synchronous velocity of the star at the present time would be well under $1 \mathrm{~km} \mathrm{~s}^{-1}$, whereas we measure $v_{1} \sin i=36 \mathrm{~km} \mathrm{~s}^{-1}$. The times $t_{\text {circ }}$ and $t_{\text {sync, } 1}$ are indicated with vertical dotted lines in Figure $10 a$, which depicts the evolution of the radius of the two stars as a function of age, for reference. The evolutionary age of the system $(0.935 \mathrm{Gyr} ; \S 5)$ is also indicated. It is seen that synchronization of the primary and circularization of the orbit are triggered by the relatively sudden increase in size suffered by the primary as it moves up the giant branch. For $t_{\mathrm{sync}, 2}$ we can only place a lower limit of $1.245 \mathrm{Gyr}$ (the same as $t_{\text {circ }}$ ) because the nuclear timescale of the secondary is $35 \%$ longer than the primary, and the evolutionary tracks do not reach sufficiently advanced stages to allow the integrations. This is again consistent with the fact that the measured $v \sin i$ of the secondary $\left(10 \mathrm{~km} \mathrm{~s}^{-1}\right)$ is much larger than the synchronous value (which is similar to the primary).

One of the characteristics of the $\lambda$ Vir system that has drawn attention in the past, particularly in connection with the Am nature of the binary, is the difference in the projected rotational velocities of the components (see, e.g., Stickland 1975). From our measurements in $\S 2.1$ the primary is rotating approximately 3.5 times more rapidly than the secondary. Both values of $v \sin i$ are low compared to the average for A-type stars in the field, which has typically been found to be the case for all Am stars. Since these objects are overwhelmingly found to be members of binary systems (Abt 1961), the connection between the slow rotation, the chemical peculiarities, and binarity has been much discussed (see, e.g., Abt \& Morrell 1995; Budaj 1996, 1997; Abt 2000; Böhm-Vitense 2006). In the following we examine extent to which differences in the evolution of the spin rates and/or differences in the evolution of the orientation of the rotation axes since formation might have contributed to the difference in the $v \sin i$ values presently observed.

The evolution of the spin of each star is a function of the changes in the moment of inertia due to evolution and also depends on the effects of tidal forces from the companion. As described above, the latter are expected to be relatively weak in the present evolutionary state of the binary, since synchronization is not expected to happen for another $300 \mathrm{Myr}(\sim 1 / 3$ of the present age of $\lambda$ Vir). To model the changes in the moment of inertia, we have made use of the Granada series of stellar evolution calculations (Claret 2004) that are well-suited for binary studies. The physics in these models is similar to that in the Yonsei-Yale models, although some of the details are somewhat different. We adopt a composition similar to that found earlier $(Z=0.01)$, and we computed mass tracks for the exact masses we measure for the stars. In Figure $10 b$ we show the change in the rotational velocity of each component with time relative to its initial value on arrival at the zero-age main sequence (ZAMS). At the present evolutionary age of the system the rotational velocity of the primary is predicted to have slowed by about $10 \%$ from its initial value, whereas the decrease for the secondary is only $2 \%-3 \%$. Given that the $v \sin i$ of the primary is currently the higher of the two, an obvious possibility is that the primary was initially rotating more rapidly than the secondary. Another is that the spin axes of the stars have different inclinations relative to the line of sight.

As indicated earlier, the timescale for alignment of the rotation axes of the binary components with the axis of the orbit due to tidal forces is typically much shorter than the timescale for circularization, and as a result, alignment is virtually always assumed. To examine whether this is actually true for $\lambda$ Vir, we consider, in addition to the differential equations of tidal evolution used above, one that describes the evolution of the orientation of the spin axis of each star, characterized by an inclination angle $i$. We follow closely the formalism by Hut (1981) with a timescale for the alignment of the spin axis with the axis of the orbit given in terms of the circularization timescale by

$$
\tau_{i}=\frac{7}{(\alpha+1)} \tau_{\text {circ }}
$$

In this expression $\alpha$ is the ratio between the orbital and rotational angular momenta, which is given by

$$
\alpha=\frac{q}{1+q}\left(\frac{a}{\beta R}\right)^{2}
$$

where $a$ is the semimajor axis of the orbit. The integrations were carried out as described above. The expected time for the alignment of the spin axis of the primary is $1.239 \mathrm{Gyr}$, not surprisingly the same as the time of synchronization. For the secondary once again we can only place a lower limit of 1.245 Gyr. It follows that neither of the rotation axes is expected to be aligned with the orbit, unless they were perfectly aligned to begin with. Therefore, the projection factor for the equatorial rotational velocities remains unknown (i.e., it is not necessarily the same as the inclination of the orbit, which we have measured precisely) and may be different for each star.

In conclusion, the measured difference in the projected rotational velocities of $\lambda$ Vir may result from the very different initial rotation rates of the two components, the different projections of 
their equatorial velocities due to misalignment of the spin axes, or perhaps to the combination of the two effects.

\section{SUMMARY AND CONCLUSION}

By combining the interferometric and radial velocity data, we have determined the three-dimensional orbit of $\lambda$ Vir to high precision. We studied the effects of bandwidth smearing on squared visibilities and closure phases. The calibration problems in the closure phases due to these effects are larger than expected, suggesting the necessity of using narrow bandwidth for precision work.

Our precise determination of the $\lambda$ Vir orbit allows us to study its physical properties accurately. We determined the masses of the two components with accuracies of $0.7 \%$ and $1.5 \%$ for the primary and the secondary, respectively. We studied the SED of $\lambda$ Vir with archival photometric and spectrophotometric data. The Kurucz model templates with $[m / \mathrm{H}]=+0.5$ fit the data best, yielding a solution with two A1 V type stars. The temperatures of the system are derived from various methods, leading to a value of $8280 \pm 200 \mathrm{~K}$ for both stars. Other properties of $\lambda \mathrm{Vir}$, such as distance, bolometric flux, luminosity, radii, and motion of the photocenter, are also determined.

The accurately determined properties allow a comparison with current stellar evolution models. The model that matches best yields a subsolar metallicity of $Z=0.0097$ and an age of $935 \mathrm{Myr}$, indicating the evolution of $\lambda$ Vir is similar to normal A stars despite their surface abundance anomalies. A study of tidal evolution in $\lambda$ Vir indicates that its orbital circularization time is $t_{\text {circ }}=$ $1.245 \mathrm{Gyr}$, larger than the present age of the system, and therefore theory agrees with the observation that the orbit is not currently circular. The predicted orbital synchronization time also implies that neither of the two stars has synchronized rotation. Furthermore, neither of the rotational axes is expected to be aligned with the orbit, implying that in addition to the possibility that the two stars have very different initial rotations, the measured differing rotational velocities may also stem from the projection of the equatorial rotational velocities.

The origin of the abundance anomalies of Am stars has been a puzzle for quite some time. It is widely believed that when stars are slow rotators, atomic diffusion will play an important role in the outer convection zones, causing abnormal abundances and therefore the Am phenomenon. However, different views have also been presented (Böhm-Vitense 2006). Although Am stars have been studied intensively, only a few of them have welldetermined properties. With its accurately determined physical properties and well-known evolution status, as well as its possibly differing rotation rates (which may lead to different diffusion efficiencies), $\lambda$ Vir is an ideal candidate for follow-up studies such as detailed abundance analyses and atomic diffusion modeling that can shed light on our understanding of the causes of the Am phenomenon.

We thank C. R. Cowley for helpful discussion and advice, and J. R. Caruso, R. J. Davis, D. W. Latham, T. Mazeh, A. A. E. Milone, R. P. Stefanik, and J. M. Zajac for obtaining most of the spectroscopic observations used in this work. We also thank the referee for a number of helpful comments. We gratefully acknowledge support for IOTA from the Smithsonian Astrophysical Observatory, the National Aeronautics and Space Administration (NASA) (for third telescope development, NNG05G1180G; for data analysis support, NNG04GI33G), and the National Science Foundation (NSF) (AST 01-38303, AST 03-52723). G. T. wishes to acknowledge partial support for this work from NSF grant AST 04-06183 and NASA's MASSIF SIM Key Project (BLF57-04). A. B. also gratefully acknowledges the support of NASA. The IONIC3 instrument has been developed by LAOG and LETI in the context of the IONIC collaboration (LAOG, IMEP, LETI). The IONIC project is funded by the CNRS and CNES (France). This research has made use of the SIMBAD database, operated at CDS, Strasbourg, France, and NASA's Astrophysics Data System Abstract Service. This publication makes use of data products from 2MASS, which is a joint project of the University of Massachusetts and the Infrared Processing and Analysis Center/ California Institute of Technology, funded by NASA and NSF. This work also makes use of services produced at the Michelson Science Center (http://msc.caltech.edu) at the California Institute of Technology.

\section{APPENDIX}

\section{OPD FLUCTUATIONS AND CLOSURE-PHASE ERRORS}

Because fringes are obtained by piezo scanning, the measured interferograms are thus temporal sequences that span several atmospheric coherence times. In our data reduction pipeline, fringe scans are divided into segments of equal time according to the atmospheric coherence time (Baldwin et al. 1996) to allow the best $\mathrm{S} / \mathrm{N}$ for averaging the closure phase. More specifically, the complex visibility of each short time segment is calculated individually for the three baselines. The triple product of each segment is thus obtained from a complex multiplication of the three visibilities. Finally, the (complex) triple products from each segment are averaged together with those from the other segments to form a single estimate of the complex triple product for each scan. The closure phase is of course derived as the phase argument of the final complex triple product (see Monnier 1999).

Figure 11 shows an example of this method. The simulated fringes are divided into segments of 16 pixels in the pipeline, corresponding to $10-20 \mathrm{~ms}$ in time (depending on the scan rate). With zero atmospheric delays, the fringe envelopes are aligned in time, and the calculation of the triple product is straightforward (and the resulting bias on the closure phase has already been discussed in $\S 3.2)$. As the atmospheric piston fluctuates and causes OPD fluctuations, the fringe envelopes may not coincide exactly in time, and thus, we must consider this additional complication on the closure-phase estimator. Because the "phase" of the fringes within the coherence envelope may not be constant with OPD, due to both source structure and due to dispersion in beam combining optics, we intuitively can see that OPD fluctuations will corrupt the measurement process. We note that this effect does not exist for the monochromatic (i.e., very narrow bandwidth) case, and we later (see eq. [A1]) derive a more quantitative bandwidth condition.

In order to investigate the influence of this effect, it is instructive to consider the case of a binary star, and we have performed simulations in this Appendix using the parameters of the $\lambda$ Vir system. We have simulated OPD fluctuations above each telescope, ensuring the OPDs are closed in triangle (i.e., $\mathrm{OPD}_{\mathrm{AB}}+\mathrm{OPD}_{\mathrm{BC}}+\mathrm{OPD}_{\mathrm{CA}}=0$ ). The resulting closure phases are then calculated using the IOTA data reduction pipeline (described above and also in $\S 2.2$ ). Figure 12 shows 20 simulated closure-phase curves for each of the two representative epochs, 2003 March 24 for piezo scan mode 1 and 2005 June 16 for mode 2 . The closure phases 


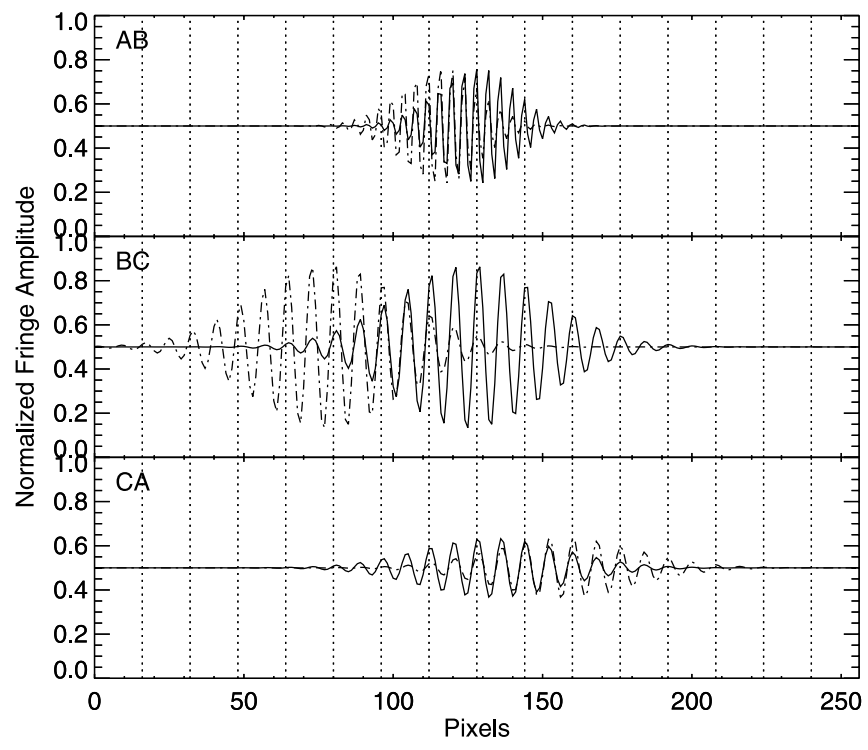

FIg. 11.- Simulated $\lambda$ Vir fringes for IOTA baselines AB, BC, and CA. The solid lines indicate normalized fringes with zero phase shift, while the dash-dotted lines show an example of fringes with phase shifts of $3,-6$, and 3 wavelengths, respectively. The vertical dotted lines divide the fringes into segments of 16 pixels.

fluctuate in both panels due to the fluctuations of extra OPDs that were assumed to follow a normal distribution with $1 \sigma$ deviation of 1.2 wavelengths (determined below). As can be seen in Figure 12, the fluctuating OPDs introduce sometimes very large errors in the closure phase (up to $\sim 10^{\circ}$ in the left panel and $\sim 30^{\circ}$ in the right) that depend on both hour angle (i.e., projected binary separation) and scan mode (which affects the interferograms). These errors are many times larger than those seen from bandwidth smearing calculated without OPD fluctuations in Figure 2, suggesting the errors from these fluctuations are dominant errors in our closure-phase measurements.

We have reduced the influence of this disturbing effect on our closure-phase modeling in $\S 3.2$ by simulating the closure-phase fluctuations for all of our data. From the scatter of simulated closure phases, we provide an estimate of the closure-phase errors to the model-fitting process. The standard deviation of the simulated OPD fluctuations was determined iteratively in model-fitting procedures in order to reduce the $\chi_{\nu}^{2}$ to close to unity. We found that just a small uncorrected atmospheric OPD fluctuation of $\pm 2 \mu$ m was enough to explain our observed closure-phase errors, and this level of OPD error is similar to actual residuals reported at IOTA by Pedretti et al. (2005).

Quite unexpectedly, we discovered that the closure-phase simulations showed "null points" where OPD fluctuations had no effect on the measured closure phases (e.g., see null fluctuation point in the right panel of Fig. 12, but not in the left panel). To look into this in more detail, we simulated the closure-phase fluctuations for both scan modes at all observed epochs. Figure 13 shows two typical epochs and the comparison of the two modes at each epoch. As we can see, the fluctuations of the two modes behave differently. Both of them have null points, but the locations of the nulls are different. This is because fringes are scanned from different sides in different modes. For example, in our case, one mode scans fringe AC from the left-hand side, while the other scans from the right-hand side, which causes the segments of fringe $\mathrm{AC}$ in the two modes to be scanned at different coherence times, thus introducing different errors to the triple products and causing the closure phases to fluctuate differently. For the case of a binary star, it is easy to prove that when any two of the three interferometric delays between components $(\boldsymbol{B} \cdot \boldsymbol{\rho})$ are equal to $\pm n / 2$ wavelengths, the closure phase will be immune to OPD fluctuations and therefore will have a null. The behaviors of other nulls may be related to their scan mode and are not yet clear. Note that these results are restricted to models of binary stars but presumably apply in general to objects with any resolved asymmetric structure affected by bandwidth smearing.
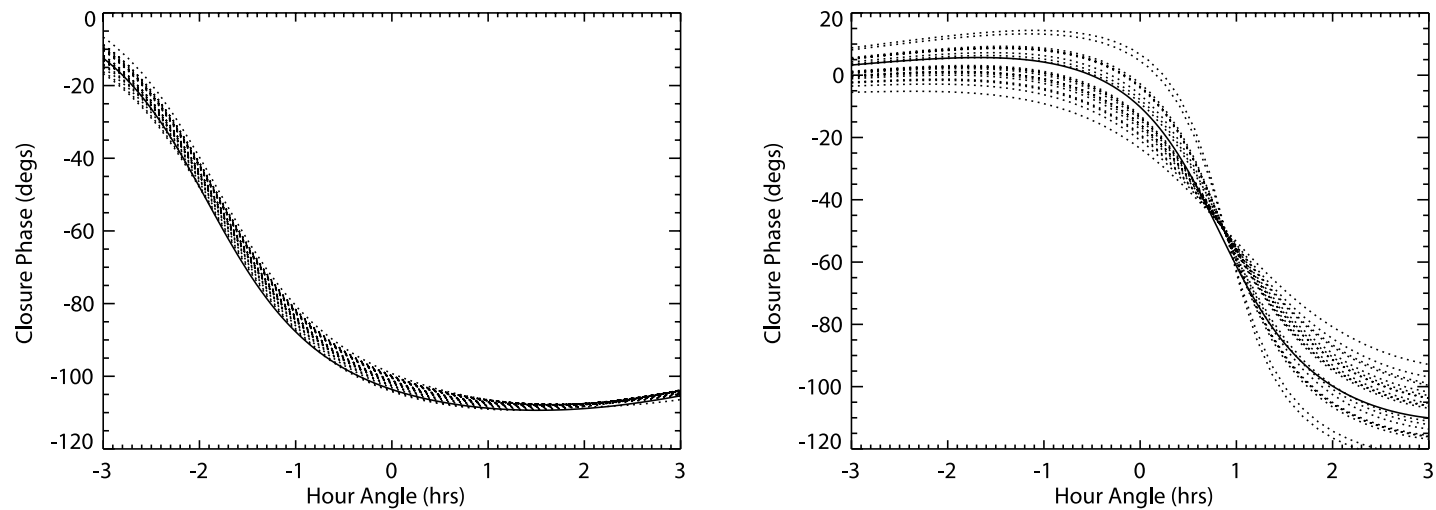

FIG. 12.-Closure-phase fluctuations due to additional OPDs caused by the varying atmospheric piston. The dotted lines indicate simulated closure phases with different OPDs. The closure-phase models with no bandwidth-smearing correction and zero OPD fluctuations are plotted as solid lines for reference. As in Fig. 2, two dates with different scan modes (left: 2003 March 24, scan mode 1; right: 2005 June 16, scan mode 2) are selected to represent the entire data. 

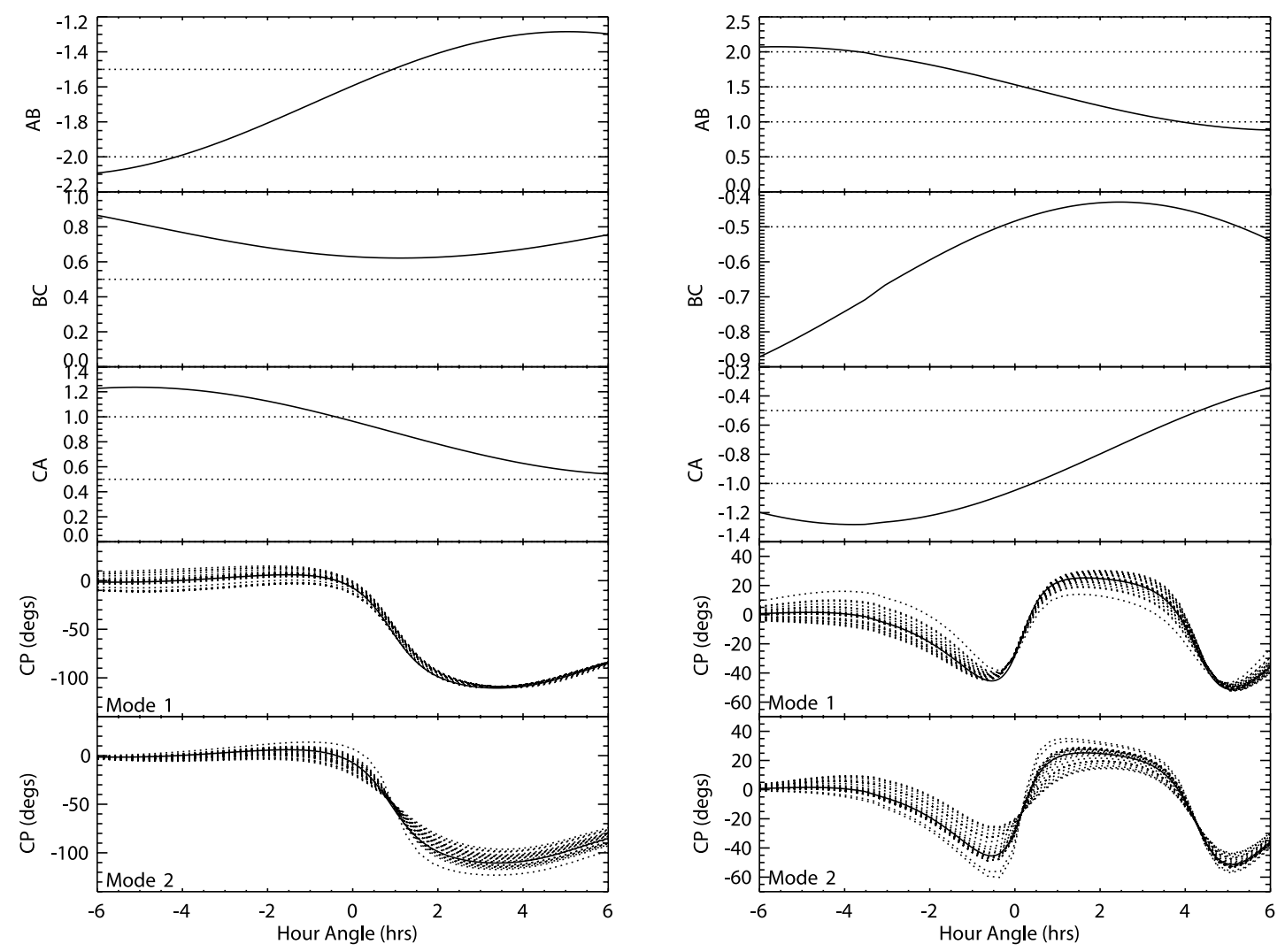

Fig. 13.-Comparison of closure-phase fluctuations between the two scan modes. We show two typical epochs at different orbital phases; the left plot is for phase 0.25 , while the right is for 0.68 . The interferometric delay $(\boldsymbol{B} \cdot \boldsymbol{\rho}$, in units of wavelengths) of the three baselines are plotted as solid lines in the top three panels of each plot. The dotted lines indicate phases of $\pm n / 2$ wavelengths, corresponding to $\pm n \pi$ radians. The two bottom panels show the corresponding closure phases for scan modes 1 and 2. The solid lines indicate the model closure phase with zero OPD fluctuation and no bandwidth-smearing correction, while the dotted lines indicate the simulated closure-phase fluctuations.

We can use our empirical study of binary stars to motivate a scaling relation for estimating when bandwidth smearing corrupts the closure-phase measurement process. In our simulations of IOTA, we found strong effects when the source structure (scale: $\rho$ ) fills $\sim \frac{1}{5}$ of the field of view defined by bandwidth smearing. Thus, we find that bandwidth smearing has a much stronger and more corruptive effect on closure phases than on visibility amplitudes. We can express this mathematically as a condition to meet in order to assure good closure-phase measurement:

$$
\frac{\boldsymbol{B} \cdot \boldsymbol{\rho}}{\lambda}<\frac{1}{5} \frac{\lambda}{\Delta \lambda}
$$

where $\boldsymbol{B} \cdot \boldsymbol{\rho}$ is the previously defined interferometric delay, $\boldsymbol{B}$ is the projected baseline vector $\left(B_{x}, B_{y}\right)$ in units of meters, and $\boldsymbol{\rho}$ is the binary angular separation $(a, b)$ in units of radians (for cases other than a binary, this represents the typical scale of source structure).

In order to avoid these closure-phase fluctuations, one could consider using a closure-phase estimator that is not affected by the fringe phase shift, e.g., an estimator that does not divide fringes into coherence segments. However, this estimator is likely to be very noisy unless the entire interferogram is scanned within a coherence time - drastically reducing the $\mathrm{S} / \mathrm{N}$ for faint objects. Since all of these problems actually stem from bandwidth smearing, we conclude that using narrow bandwidth is a better approach and is of importance for precision work.

Abt, H. A. 1961, ApJS, 6, 37 2000, ApJ, 544,933

Abt, H. A., \& Morrell, N. I. 1995, ApJS, 99, 135

Baldwin, J. E., et al. 1996, A\&A, 306, L13

Balona, L. A. 1994, MNRAS, 268, 119

Berger, J.-P., et al. 2003, Proc. SPIE, 4838, 1099

Blackwell, D. E., Petford, A. D., Arribas, S., Haddock, D. J., \& Selby, M. J. 1990, A\&A, 232, 396

Boden, A. F. 2000, in Principles of Long Baseline Interferometry, ed. P. R.

Lawson (Pasadena: NASA), 9

Böhm-Vitense, E. 2006, PASP, 118, 419

Breger, M. 1976, ApJS, 32, 7

Budaj, J. 1996, A\&A, 313, 523 1997, A\&A, 326, 655

\section{REFERENCES}

Burnashev, V. I. 1985, Abastumanskaia Astrofiz. Obs. Bull., 59, 83

Campbell, W. W., Moore, J. H., Wright, W. H., \& Duncan, J. C. 1911, Lick Obs. Bull., 6, 140

Cayrel, R., Burkhart, C., \& van't Veer, C. 1991, in IAU Symp. 145, Evolution of Stars: the Photospheric Abundance Connection, ed. G. Michaud \& A. V. Tutukov (Dordrecht: Kluwer), 99

Claret, A. 2004, A\&A, 424, 919

Claret, A., \& Cunha, N. C. S. 1997, A\&A, 318, 187

Colacevich, A. 1941, Oss. Mem. Arcetri, 58, 28

Coude du Foresto, V., Ridgway, S., \& Mariotti, J.-M. 1997, A\&AS, 121, 379

Cowley, A., Cowley, C., Jaschek, M., \& Jaschek, C. 1969, AJ, 74, 375

Cox, A. N., ed. 2000, Allen's Astrophysical Quantities (4th ed.; New York: AIP)

Crawford, D. L. 1979, AJ, 84, 1858 
Demarque, P., Woo, J.-H., Kim, Y.-C., \& Yi, S. K. 2004, ApJS, 155, 667

Gray, D. F. 1992, Science, 257, 1978

Griffin, R. E. 2002, AJ, 123, 988

Hummel, C. A., Carquillat, J.-M., Ginestet, N., Griffin, R. F., Boden, A. F., Hajian, A. R., Mozurkewich, D., \& Nordgren, T. E. 2001, AJ, 121, 1623

Hut, P. 1981, A\&A, 99, 126

Kunzli, M., North, P., Kurucz, R. L., \& Nicolet, B. 1997, A\&AS, 122, 51

Kurucz, R. L., Peytremann, E., \& Avrett, E. H. 1974, Blanketed Model Atmospheres for Early-Type Stars (Washington: Smithsonian Inst.), 37

Latham, D. W., Mathieu, R. D., Milone, A. A. E., \& Davis, R. J. 1992, in IAU Symp. 151, Evolutionary Processes in Interacting Binary Stars, ed. Y. Kondo, R. Sistero, \& R. S. Polidan (Dordrecht: Kluwer), 471

Latham, D. W., Nordstroem, B., Andersen, J., Torres, G., Stefanik, R. P., Thaller, M., \& Bester, M. J. 1996, A\&A, 314, 864

Latham, D. W., Stefanik, R. P., Torres, G., Davis, R. J., Mazeh, T., Carney, B. W., Laird, J. B., \& Morse, J. A. 2002, AJ, 124, 1144

Lejeune, T., Cuisinier, F., \& Buser, R. 1998, A\&AS, 130, 65

Levato, A. 1975, A\&AS, 19, 91

Mermilliod, J.-C., Mermilliod, M., \& Hauck, B. 1997, A\&AS, 124, 349

Michaud, G. 1980, AJ, 85, 589

Michaud, G., Richer, J., \& Richard, O. 2005, ApJ, 623, 442

Monnier, J. D. 1999, in Principles of Long Baseline Interferometry, ed. P. R. Lawson (Pasadena: NASA), 203

Monnier, J. D., et al. 2004, ApJ, 602, L57 2006, ApJ, 647, 444

Moon, T. T., \& Dworetsky, M. M. 1985, MNRAS, 217, 305

Napiwotzki, R., Schoenberner, D., \& Wenske, V. 1993, A\&A, 268, 653

Nordström, B., Latham, D. W., Morse, J. A., Milone, A. A. E., Kurucz, R. L., Andersen, J., \& Stefanik, R. P. 1994, A\&A, 287, 338

Pedretti, E., et al. 2004, PASP, 116, 377 2005, Appl. Opt., 44, 5173

Popper, D. M. 1980, ARA\&A, 18, 115
Pourbaix, D., \& Jorissen, A. 2000, A\&AS, 145, 161

Press, W. H., Teukolsky, S. A., Vetterling, W. T., \& Flannery, B. P. 1992, in Numerical Recipes in, C. The Art of Scientific Computing (2nd ed.; Cambridge: Cambridge Univ. Press)

Richer, J., Michaud, G., Rogers, F., Iglesias, C., Turcotte, S., \& LeBlanc, F. 1998, ApJ, 492, 833

Richer, J., Michaud, G., \& Turcotte, S. 2000, ApJ, 529, 338

Roby, S. W., \& Lambert, D. L. 1990, ApJS, 73, 67

Sadakane, K., \& Okyudo, M. 1989, PASJ, 41, 1055

Shorlin, S. L. S., Wade, G. A., Donati, J.-F., Landstreet, J. D., Petit, P., Sigut, T. A. A., \& Strasser, S. 2002, A\&A, 392, 637

Smalley, B., \& Dworetsky, M. M. 1995, A\&A, 293, 446

Stefanik, R. P., Latham, D. W., \& Torres, G. 1999, in IAU Colloq. 170, Precise Stellar Radial Velocities, ed. J. B. Hearnshaw \& C. D. Scarfe (ASP Conf. Ser. 185; San Francisco: ASP), 354

Stickland, D. J. 1975, in The Physics of Ap Stars, ed. W. W. Weiss, H. Jenkner, \& H. J. Wood (Vienna: Univ. Wien), 701 1990, Observatory, 110, 43

Titus, J., \& Morgan, W. W. 1940, ApJ, 92, 256

Torres, G. 2006, AJ, 131, 1022

Torres, G., Andersen, J., Nordström, B., \& Latham, D. W. 2000, AJ, 119, 1942

Torres, G., Neuhäuser, R., \& Guenther, E. W. 2002, AJ, 123, 1701

Torres, G., Stefanik, R. P., Andersen, J., Nordstrom, B., Latham, D. W., \& Clausen, J. V. 1997, AJ, 114, 2764

Traub, W. A., et al. 2003, Proc. SPIE, 4838, 45

van Leeuwen, F., \& Evans, D. W. 1998, A\&AS, 130, 157

van't Veer-Menneret, C., Coupry, M. F., \& Burkhart, C. 1988, A\&A, 203, 123

Yi, S., Demarque, P., Kim, Y.-C., Lee, Y.-W., Ree, C. H., Lejeune, T., \& Barnes, S. 2001, ApJS, 136, 417

Zahn, J.-P. 1977, A\&A, 57, 383 1989, A\&A, 220, 112

Zucker, S., \& Mazeh, T. 1994, ApJ, 420, 806 